\title{
Bootstrapping Unit Root Tests with Covariates ${ }^{1}$
}

\author{
Yoosoon Chang \\ Department of Economics \\ Rice University \\ Robin C. Sickles \\ Department of Economics \\ Rice University \\ Wonho Song \\ Department of Economics \\ Rice University
}

\begin{abstract}
We consider the bootstrap method for the covariates augmented DickeyFuller (CADF) unit root test suggested in Hansen (1995) which uses related variables to improve the power of univariate unit root tests. It is shown that there are substantial power gains from including correlated covariates. The limit distribution of the CADF test, however, depends on the nuisance parameter that represents the correlation between the equation error and the covariates. Hence, inference based directly on the CADF test is not possible. To provide a valid inferential basis for the CADF test, we propose to use the bootstrap procedure to obtain critical values, and establish the asymptotic validity of the bootstrap CADF test. Simulations show that the bootstrap CADF test significantly improves the finite sample size performances of the CADF test, especially when the covariates are highly correlated with the error. Indeed, the bootstrap CADF test offers drastic power gains over the conventional ADF test. We apply our testing procedures to the extended Nelson-Plosser data set for the post-1929 samples as well as postwar annual CPI-based real exchange rates for 14 OECD countries.
\end{abstract}

This version: September 18, 2001

JEL Classification: C12, C15, C22.

Key words and phrases: Unit root tests, covariates, bootstrap consistency.

\footnotetext{
${ }^{1}$ We are grateful to Joon Park, Bill Brown, and David Papell for helpful discussions and comments. Correspondence address to: Robin Sickles, Department of Economics - MS 22, Rice University, 6100 Main Street, Houston, TX 77005-1892, Tel: 713-348-3322, Fax: 713-348-5278, Email: rsickles@rice.edu.
} 


\section{Introduction}

Conventional univariate tests for the presence of unit roots in aggregate economic time series have important implications for the conduct of domestic macro and international economic policy. These tests unfortunately have been plagued by reliance on relatively short time series with relatively low frequencies. Size distortions and low power are wellknown problems with conventional testing procedures (see, e.g., Stock 1991, and Campbell and Perron 1991, Domowitz and El-Gamal, 2001). Current macroeconomic theory provides little in the way of guidance on how to increase the power and moderate size distortions other than by increasing the length of the time series. Reliance on the sort of information that was utilized in conventional empirical macroeconomics before the Lucas critique took hold, namely the information contained in the correlated errors of other overidentified equations in the structural system, has little apparent place in the current unit root testing literature. Even agreement on the candidate set of correlated series has little theoretical basis (Stock and Watson, 1999). The first wide-spread use of univariate tests for the presence of unit roots was carried in the seminal work of Nelson and Plosser (1982) who found that most U.S. macroeconomic time series could be characterized as a univariate time series structure with a unit root. Subsequent empirical analyses have largely confirmed their findings while the literature continues to acknowledge the low power of unit root tests and an implication of this low power, that in finite samples it is almost impossible to discriminate between a unit root process and one which is very close to it.

Clearly the unit root hypothesis has important implications for determining the effect of random shocks on an economic system and the literature has not been silent on the many efforts to overcome the low power of conventional unit root tests. One such contribution was made by Hansen (1995) who noted that conventional univariate unit root tests ignore potentially useful information from related time series and that the inclusion of related stationary covariates in the regression equation may lead to a more precise estimate of the autoregressive coefficient. He proposed to use the covariates augmented Dickey-Fuller $(\mathrm{CADF})$ unit root test rather than conventional univariate unit root tests. He analyzed the asymptotic local power functions for the CADF $t$-statistic and discovered that enormous power gains could be achieved by the inclusion of appropriate covariates. His Monte Carlo study suggested that such gains were also possible in the finite sample power performances of the CADF vis-a-vis conventional ADF test.

Hansen showed that the limit distribution of the CADF test is dependent on the nuisance parameter that characterizes the correlation between the equation error and the covariates. Therefore, it is not possible to perform valid statistical inference directly using the CADF test. To deal with this inferential difficulty, Hansen (1995) suggested using critical values based on an estimated nuisance parameter. ${ }^{2}$ His two-step procedure can be a practical solution for the implementation of the CADF test. Strictly speaking, however, the validity of the resulting test is questionable since the variability in the nuisance parameter estimate is ignored.

\footnotetext{
${ }^{2}$ Hansen (1995)'s Table 1 provides asymptotic critical values for the CADF $t$-statistic for values of the nuisance parameter in steps of .1 via simulations. For intermediate values of the nuisance parameter, critical values are selected by interpolation.
} 
In this paper, we apply the bootstrap method to the CADF test to deal with the nuisance parameter dependency and to provide a valid basis for inference based on the CADF test. In particular, we show the consistency of the bootstrap CADF test and establish the asymptotic validity of the critical values from the bootstrap distribution of the test. The finite sample performances of the bootstrap CADF test are investigated and compared with those of the CADF test and the usual ADF test through simulations. The simulations show that the CADF test based on the two-step procedure suffers serious size distortions, especially when the covariates are highly correlated with the error, while our bootstrap CADF test significantly improves the finite sample size performances of the CADF test. Moreover, the bootstrap CADF test offers dramatic power gains over the conventional ADF test.

As an illustration, we apply our covariate tests and standard unit root tests in a reexamination of the stationarity of U.S. domestic macroeconomic aggregates and international rates of exchange. The former are analyzed with the extended Nelson-Plosser data set for the post-1929 period. We investigate whether the findings of unit roots in the Nelson-Plosser data set are reversed when the more powerful covariate tests are used. The latter are examined using postwar annual CPI-based real exchange rates for 14 OECD countries, for which most previous studies failed to reject the null hypothesis of a unit root. We find that our new covariate test rejects the unit root hypothesis in all the series in the Nelson-Plosser data set for the period 1930-1988 and in most cases for the postwar real exchange rates.

The paper is organized as follows. Section 2 introduces the unit root test with covariates and derives limit theories for the sample tests. Section 3 applies the bootstrap methodology to the sample tests considered in Section 2 and establishes the asymptotic validity of the bootstrap test. Section 3 also provides a discussion of practical issues arising from the implementation of the bootstrap methodology. In Section 4, we conduct simulations to investigate the finite sample performances of the bootstrap CADF test. Empirical applications are presented in Section 5 while Section 6 concludes. All mathematical proofs are provided in the Appendix.

\section{Unit Root Tests with Covariates}

\subsection{Model and Assumptions}

We consider the time series $\left(y_{t}\right)$ given by

$$
\triangle y_{t}=\alpha y_{t-1}+u_{t}
$$

for $t=1, \ldots, n$, where $\triangle$ is the usual difference operator. We let the regression errors $\left(u_{t}\right)$ in the model (1) to be serially correlated, and also allow them to be related to other stationary covariates. To define the data generating process for the errors $\left(u_{t}\right)$ more explicitly, let $\left(w_{t}\right)$ be an $m$-dimensional stationary covariates. It is assumed that the errors $\left(u_{t}\right)$ are given by a $p$-th order autoregressive (AR) process specified as

$$
\alpha(L) u_{t}=\beta(L)^{\prime} w_{t}+\varepsilon_{t}
$$

where $L$ is the lag operator, $\alpha(z)=1-\sum_{k=1}^{p} \alpha_{k} z^{k}$ and $\beta(z)=\sum_{k=-r}^{q} \beta_{k} z^{k}$. 
We consider the test of the unit root null hypothesis $\alpha=0$ for $\left(y_{t}\right)$ given as in (1), against the alternative of the stationarity $\alpha<0$. The initial value of $y_{0}$ of $\left(y_{t}\right)$ does not affect our subsequent analysis so long as it is stochastically bounded, and therefore we set it at zero for expositional brevity.

Under the null hypothesis of unit root, $\triangle y_{t}=u_{t}$ and we have from (2) that

$$
\triangle y_{t}=\alpha y_{t-1}+\sum_{k=1}^{p} \alpha_{k} \Delta y_{t-k}+\sum_{k=-r}^{q} \beta_{k}^{\prime} w_{t-k}+\varepsilon_{t}
$$

which is an autoregression of $\triangle y_{t}$ augmented by its lagged level $y_{t-1}$ and the leads and lags of the $m$ stationary covariates in $w_{t}$. Indeed, the above regression may be viewed as a further augmentation of the usual ADF regression, which is an autoregression of $\triangle y_{t}$ augmented by its lagged level $y_{t-1}$ only. Our test statistics for testing the unit root in $\left(y_{t}\right)$, which are introduced in the next section, will be based on the least squares estimator for $\alpha$ from this CADF regression.

For the subsequent analysis, we also need to be more explicit about the data generating process for the stationary variables $\left(w_{t}\right)$ that are used as covariates. We assume that $\left(w_{t}\right)$ is generated by an $\operatorname{AR}(\ell)$ process as

$$
\Phi(L) w_{t+r+1}=\eta_{t}
$$

where $\Phi(z)=I_{m}-\sum_{k=1}^{\ell} \Phi_{k} z^{k}$.

To define explicitly the correlation between the covariates $\left(w_{t}\right)$ and the series to be tested $\left(y_{t}\right)$, we consider together the innovations $\left(\eta_{t}\right)$ and $\left(\varepsilon_{t}\right)$ that generate, respectively, the covariates $\left(w_{t}\right)$ and the regression error $\left(u_{t}\right)$, which in turn generates $\left(y_{t}\right)$. Define

$$
\xi_{t}=\left(\varepsilon_{t}, \eta_{t}^{\prime}\right)^{\prime}
$$

and denote by $|\cdot|$ the Euclidean norm: for a vector $x=\left(x_{i}\right),|x|^{2}=\sum_{i} x_{i}^{2}$ and for a matrix $A=\left(a_{i j}\right),|A|=\sum_{i, j} a_{i j}^{2}$. We now lay out assumptions needed for the development of our asymptotic theory.

Assumption 2.1 We assume

(a) Let $\left(\xi_{t}\right)$ be a sequence of iid random variables such that $\mathbf{E} \xi_{t}=0, \mathbf{E} \xi_{t} \xi_{t}^{\prime}=\Sigma>0$ and $\mathbf{E}\left|\xi_{t}\right|^{s}<\infty$ for some $s \geq 4$.

(b) $\alpha(z), \operatorname{det}(\Phi(z)) \neq 0$ for all $|z| \leq 1$.

Here, we assume $\left(\xi_{t}\right)$ to be an iid sequence, which is stronger than needed, to make the bootstrap procedure in the next section meaningful. Assumption 2.1 (a) states that the regression error $\left(\varepsilon_{t}\right)$ in equation (3) is serially uncorrelated and independent of $\eta_{t+k}$ for $k \geq 1$. The condition effectively implies that the regression error $\varepsilon_{t}$ is orthogonal to the lagged differences of the dependent variable $\left(\triangle y_{t-1}, \ldots, \triangle y_{t-p}\right)$ and the leads and lags of the stationary covariates $\left(w_{t+r}, \ldots, w_{t-q}\right)$, which is necessary for the regression (3) to be consistently estimated via usual least squares estimation. Such orthogonalities can be achieved by appropriately increasing the orders $p, q$ and $r$ in the lag polynomials $\alpha(L)$ and $\beta(L)$ as the sample size tends to infinity, as shown in Saikkonen (1991). To discuss 
it more explicitly, let $v_{t}=\sum_{k=-r}^{q} \beta_{k}^{\prime} w_{t-k}+\varepsilon_{t}$. The error $v_{t}$ becomes orthogonal to the lagged differences $\left(\triangle y_{t-1}, \ldots, \triangle y_{t-p}\right)$ if we allow the order $p$ in the lag polynomial $\alpha(L)$ in (2) to increase at a controlled rate as the sample size increases. The orthogonality between the error $\varepsilon_{t}$ in $v_{t}$ and the leads and lags of the stationary covariates $\left(w_{t+r}, \ldots, w_{t-q}\right)$ can also be achieved if the orders $q$ and $r$ in the lag polynomial $\beta(L)$ are sufficiently large enough to whiten the error. Moreover, we may extend the analysis to allow $\alpha(L)$ to be an infinite order lag polynomial and approximate it by a finite order AR with the order increasing with the sample size, as done by Berk (1974), Said and Dickey (1984) and more recently by Chang and Park (2001). Our subsequent theory will also hold under these schemes. As $q$ and $r$ increase, the error $\varepsilon_{t}$ will become independent of the innovation $\eta_{t}$ at all leads and lags, and as $p$ increases $\varepsilon_{t}$ becomes orthogonal to the lagged differences of the dependent variable. Hence, it is natural to think of the error $\varepsilon_{t}$ as a residual, i.e., $\alpha_{k}$ 's and $\beta_{k}$ 's are the coefficients such that $\varepsilon_{t}$ is orthogonal to all of the included regressors $\left(\triangle y_{t-1}, \ldots, \triangle y_{t-p} ; w_{t+r}, \ldots, w_{t-q}\right)$.

Under Assumption 2.1 (a), the following invariance principle holds

$$
\frac{1}{\sqrt{n}} \sum_{t=1}^{[n s]} \xi_{t} \rightarrow_{d} B(s)
$$

for $s \in[0,1]$ as $n \rightarrow \infty$. The limit process $B=\left(B_{\varepsilon}, B_{\eta}^{\prime}\right)^{\prime}$ is an $(1+m)$-dimensional vector Brownian motion with covariance matrix

$$
\Sigma=\left(\begin{array}{cc}
\sigma_{\varepsilon}^{2} & \sigma_{\varepsilon \eta} \\
\sigma_{\eta \varepsilon} & \Sigma_{\eta}
\end{array}\right)
$$

The asymptotic behavior of $\left(y_{t}\right)$ is determined by that of $\left(u_{t}\right)$ as shown in model (1), and the latter is dependent upon the limiting behaviors of the stationary covariates $\left(w_{t}\right)$ and the innovations $\left(\varepsilon_{t}\right)$ as indicated in the relation (2). We may then derive the limit behavior of $\left(u_{t}\right)$ using the specification given in (2) from those of $\left(\varepsilon_{t}\right)$ and $\left(w_{t}\right)$ as follows:

$$
\frac{1}{\sqrt{n}} \sum_{t=1}^{[n s]} u_{t} \rightarrow_{d} \pi(1)\left(\beta(1)^{\prime} \Psi(1) B_{\eta}(s)+B_{\varepsilon}(s)\right)
$$

as $n \rightarrow \infty$, where $\pi(1)=1 / \alpha(1)$ and $\Psi(1)=\Phi(1)^{-1}$. This is derived in Lemma A.1 (b) in Appendix. The variance of the limit process given in the previous equation is easily derived as

$$
\sigma_{u}^{2}=\pi(1)^{2}\left(\beta(1)^{\prime} \Psi(1) \Sigma_{\eta} \Psi(1)^{\prime} \beta(1)+\sigma_{\varepsilon}^{2}+2 \beta(1)^{\prime} \Psi(1) \sigma_{\eta \varepsilon}\right)
$$

using the parameters defined in the preceding equations.

Let $z_{t}=\left(\triangle y_{t-1}, \ldots, \triangle y_{t-p}, w_{t+r}^{\prime}, \ldots, w_{t-q}^{\prime}\right)^{\prime}$. We assume

Assumption $2.2 \sigma_{u}^{2}>0$ and $\mathbf{E} z_{t} z_{t}^{\prime}>0$.

The condition $\sigma_{u}^{2}>0$ ensures that the series $\left(y_{t}\right)$ is $\mathrm{I}(1)$ when $\alpha=0$, which is necessary to be able to interpret testing $\alpha=0$ as testing for a unit root in $\left(y_{t}\right)$. The condition $\mathbf{E} z_{t} z_{t}^{\prime}>0$ implies that the stationary regressors in $z_{t}$ are asymptotically linearly independent, which is 
required along with the condition Assumption 2.1 (a) for the consistency of the LS coefficient estimates for $\left(z_{t}\right){ }^{3}$

\subsection{Covariates Augmented Unit Root Tests}

To introduce our test statistics more effectively, we first define

$$
\begin{aligned}
A_{n} & =\sum_{t=1}^{n} y_{t-1} \varepsilon_{t}-\left(\sum_{t=1}^{n} y_{t-1} z_{t}^{\prime}\right)\left(\sum_{t=1}^{n} z_{t} z_{t}^{\prime}\right)^{-1}\left(\sum_{t=1}^{n} z_{t} \varepsilon_{t}\right) \\
B_{n} & =\sum_{t=1}^{n} y_{t-1}^{2}-\left(\sum_{t=1}^{n} y_{t-1} z_{t}^{\prime}\right)\left(\sum_{t=1}^{n} z_{t} z_{t}^{\prime}\right)^{-1}\left(\sum_{t=1}^{n} z_{t} y_{t-1}\right) \\
C_{n} & =\sum_{t=1}^{n} \varepsilon_{t}^{2}-\left(\sum_{t=1}^{n} \varepsilon_{t} z_{t}^{\prime}\right)\left(\sum_{t=1}^{n} z_{t} z_{t}^{\prime}\right)^{-1}\left(\sum_{t=1}^{n} z_{t} \varepsilon_{t}\right) .
\end{aligned}
$$

Now we have

$$
\begin{aligned}
\hat{\alpha}_{n} & =A_{n} B_{n}^{-1} \\
\hat{\sigma}_{n}^{2} & =n^{-1}\left(C_{n}-A_{n}^{2} B_{n}^{-1}\right) \\
s\left(\hat{\alpha}_{n}\right)^{2} & =\hat{\sigma}_{n}^{2} B_{n}^{-1}
\end{aligned}
$$

where $\hat{\alpha}_{n}$ is the OLS estimator of $\alpha$ from the covariates augmented regression (3), $\hat{\sigma}_{n}^{2}$ is the usual error variance estimator, and $s\left(\hat{\alpha}_{n}\right)$ is the estimated standard error for $\hat{\alpha}_{n}$. We also let

$$
\hat{\alpha}_{n}(1)=1-\sum_{k=1}^{p} \hat{\alpha}_{k}
$$

where $\hat{\alpha}_{k}$ 's are the OLS estimators of $\alpha_{k}$ 's in the CADF regression (3).

The statistics that we will consider in the paper are given by

$$
\begin{aligned}
S_{n} & =\frac{n \hat{\alpha}_{n}}{\hat{\alpha}_{n}(1)} \\
T_{n} & =\frac{\hat{\alpha}_{n}}{s\left(\hat{\alpha}_{n}\right)} .
\end{aligned}
$$

Note that $S_{n}$ is a test for the unit root based on the estimated unit root regression coefficient, and $T_{n}$ is the usual $t$-statistics for testing the unit root hypothesis from the CADF regression (3). The test $T_{n}$ is considered in Hansen (1995).

The limit theories for the tests $S_{n}$ and $T_{n}$ are given in

\footnotetext{
${ }^{3}$ As discussed below Assumption 2.1, the entire stationary regressors $z_{t}$ in the regression (3) are orthogonal to the regression error $\varepsilon_{t}$, i.e., $\mathbf{E} z_{t} \varepsilon_{t}=0$ under Assumption 2.1 (a).
} 
Theorem 2.3 Under Assumptions 2.1 and 2.2, we have

$$
\begin{aligned}
& S_{n} \rightarrow d \quad \sigma_{\varepsilon} \frac{\int_{0}^{1} Q(s) d P(s)}{\int_{0}^{1} Q(s)^{2} d s} \\
& T_{n} \rightarrow d \frac{\int_{0}^{1} Q(s) d P(s)}{\left(\int_{0}^{1} Q(s)^{2} d s\right)^{1 / 2}}
\end{aligned}
$$

as $n \rightarrow \infty$, where

$$
Q(s)=\beta(1)^{\prime} \Psi(1) B_{\eta}(s)+B_{\varepsilon}(s)
$$

and $P(s)=B_{\varepsilon}(s) / \sigma_{\varepsilon}$.

The asymptotic distributions are presented explicitly in terms of the Brownian motions $B_{\varepsilon}$ and $B_{\eta}$ via $Q=\beta(1)^{\prime} \Psi(1) B_{\eta}+B_{\varepsilon}$ and $P=B_{\varepsilon} / \sigma_{\varepsilon}$. In this way we can easily relate the asymptotic distributions of the bootstrapped tests, which are developed in the next section, to the limit distributions of the sample statistics given above.

The null asymptotic distribution for the CADF test $T_{n}$ given in Theorem 2.3 is actually equivalent to the one derived in Hansen (1995, Theorem 3). To deal with the nuisance parameter dependency, the limit distributions there are, however, derived from the limit Brownian motions $\left(B_{\varepsilon}, B_{v}\right)^{\prime}$ for the partial sum processes of $\left(\varepsilon_{t}, v_{t}\right)^{\prime}$ where $v_{t}=\beta(L)^{\prime} w_{t}+\varepsilon_{t}$. Notice that $B_{v}=Q$, where $Q$ is defined in Theorem 2.3 above. The limit distributions are then presented in terms of the standard Brownian motions defined from the Brownian motions $B_{\varepsilon}$ and $B_{v}$. To see this, let $U$ and $W$ be independent standard Brownian motions. Then we may express the limit Brownian motion $\left(B_{\varepsilon}, B_{v}\right)^{\prime}=\left(B_{\varepsilon}, Q\right)^{\prime}$ as

$$
\left(\begin{array}{c}
B_{\varepsilon} \\
Q
\end{array}\right)=\left(\begin{array}{c}
\sigma_{\varepsilon} \sqrt{1-\rho^{2}} U+\sigma_{\varepsilon v} W / \sigma_{v} \\
\sigma_{v} W
\end{array}\right)
$$

with $\sigma_{v}^{2}=\sigma_{u}^{2} / \pi(1)^{2}, \sigma_{v \varepsilon}=\beta(1)^{\prime} \Psi(1) \sigma_{\eta \varepsilon}+\sigma_{\varepsilon}^{2}$, and $\rho^{2}=\sigma_{v \varepsilon}^{2} /\left(\sigma_{\varepsilon}^{2} \sigma_{v}^{2}\right)$, where the nuisance parameters are obtained from (6) and (7). We may further simplify $B_{\varepsilon}$ as

$$
B_{\varepsilon}=\sigma_{\varepsilon}\left(\sqrt{1-\rho^{2}} U+\rho W\right) .
$$

Let $P=B_{\varepsilon} / \sigma_{\varepsilon}=\left(\sqrt{1-\rho^{2}} U+\rho W\right)$ and $Q=\sigma_{v} W$. Then, the null asymptotics for $T_{n}$ derived in Theorem 2.3 becomes

$$
T_{n} \rightarrow d \rho \frac{\int_{0}^{1} W d W}{\left(\int_{0}^{1} W^{2}\right)^{1 / 2}}+\sqrt{1-\rho^{2}} \frac{\int_{0}^{1} W d U}{\left(\int_{0}^{1} W^{2}\right)^{1 / 2}} .
$$

Notice that the limit Brownian motion $U$ is independent of $W$, and consequently the distribution of the stochastic integral $\int_{0}^{1} W d U$ is mixed normal with the mixing variate $\int_{0}^{1} W^{2}$. It 
is now easy to see that the limit distribution obtained in the previous equation is equivalent to the limit distribution derived in Hansen (1995).

The asymptotic distributions for both $S_{n}$ and $T_{n}$ are nonstandard and depend upon the nuisance parameters that characterize the correlation between the covariates and the regression error. The limit distributions are therefore basically unknown. Consequently it is impossible to perform valid statistical inference based directly on the CADF tests. As a feasible practical solution, one may simulate critical values for the tests for each value of the nuisance parameter and use its estimated value to obtain the most appropriate critical value available from the tabulated values. ${ }^{4}$ This two-step procedure can be a feasible practical solution for the implementation of the CADF tests; however, the resulting tests will not be valid in strict sense since the variability in the nuisance parameter estimate is not properly taken into account.

The models with deterministic components can be analyzed similarly. When the time series $\left(z_{t}\right)$ with a nonzero mean is given by

$$
z_{t}=\mu+y_{t}
$$

or with a linear time trend

$$
z_{t}=\mu+\delta t+y_{t}
$$

where $\left(y_{t}\right)$ is generated as in (1), we may test for the presence of the unit root in the process $\left(y_{t}\right)$ from the CADF regression (3) defined with the fitted values $\left(y_{t}^{\mu}\right)$ or $\left(y_{t}^{\tau}\right)$ obtained from the preliminary regression (12) or (13). The limit theories for the CADF tests given in Theorem 2.3 extend easily to the models with nonzero mean and deterministic trends, and are given similarly with the following demeaned and detrended Brownian motions

$$
Q^{\mu}(s)=Q(s)-\int_{0}^{1} Q(t) d t
$$

and

$$
Q^{\tau}(s)=Q(s)+(6 s-4) \int_{0}^{1} Q(t) d t-(12 s-6) \int_{0}^{1} t Q(t) d t
$$

in the place of the Brownian motion $Q(s)$.

In the next section, we consider bootstrapping the covariates augmented tests $S_{n}$ and $T_{n}$ to deal with the nuisance parameter dependency problem and to provide a valid basis for inference based on the covariates augmented unit root tests.

\section{Bootstrap Unit Root Tests with Covariates}

In this section, we consider the bootstrap for the covariates augmented unit root tests $S_{n}$ and $T_{n}$ introduced in the previous section. We establish the bootstrap consistency of the

\footnotetext{
${ }^{4}$ Noting that the null limit distribution of the CADF $t$-test depends only on the correlation coefficient $\rho^{2}$, Hansen (1995, Table 1, p.1155) provides the asymptotic critical values for the CADF $t$-test for values of $\rho^{2}$ from 0.1 to 1 in steps of 0.1 . The estimate for $\rho^{2}$ is constructed as $\hat{\rho}^{2}=\hat{\sigma}_{v \varepsilon}^{2} / \hat{\sigma}_{v}^{2} \hat{\sigma}_{\varepsilon}^{2}$, where $\hat{\sigma}_{v \varepsilon}, \hat{\sigma}_{v}^{2}$ and $\hat{\sigma}_{\varepsilon}^{2}$ are consistent nonparametric estimators of the corresponding parameters.
} 
tests and show the asymptotic validity of the tests. Throughout the paper, we use the usual notation $*$ to signify the bootstrap samples, and use $\mathbf{P}^{*}$ and $\mathbf{E}^{*}$ respectively to denote the probability and expectation conditional on a realization of the original sample. Various issues arising in practical implementation of the bootstrap methodology are also addressed.

To construct the bootstrap CADF tests, we first generate the bootstrap samples for the $m$-dimensional stationary covariates $\left(w_{t}\right)$ and the series $\left(y_{t}\right)$ to be tested. We begin by constructing the fitted residuals which will be used as the basis for generating the bootstrap samples. We first let $u_{t}=\Delta y_{t}$ and fit the regression

$$
u_{t}=\sum_{k=1}^{p} \tilde{\alpha}_{k} u_{t-k}+\sum_{k=-r}^{q} \tilde{\beta}_{k}^{\prime} w_{t-k}+\tilde{\varepsilon}_{t}
$$

by the usual OLS regression. It is important to base the bootstrap sampling on regression (14) with the unit root restriction $\alpha=0$ imposed. The samples generated by regression (3) without the unit root restriction do not behave like unit root processes, and this will render the subsequent bootstrap procedures inconsistent as shown in Basawa et al. (1991).

Next, we fit the $\ell$-th order autoregression of $w_{t}$ as

$$
w_{t+r+1}=\tilde{\Phi}_{1} w_{t+r}+\cdots+\tilde{\Phi}_{\ell} w_{t+r-\ell+1}+\tilde{\eta}_{t}
$$

by the usual OLS regression. We may prefer, especially in small samples, to use the YuleWalker method to estimate (15) since it always yields an invertible autoregression, thereby ensuring the stationarity of the process $w_{t}$ [see, e.g., Brockwell and Davis (1991, Sections 8.1 and 8.2)]. As the sample size increases, however, the problem of noninvertibility in the OLS estimation vanishes a.s., and the two methods become equivalent. Our subsequent results are applicable also for the Yule-Walker method, since it is asymptotically equivalent to the OLS method.

We then generate the $(1+m)$-dimensional bootstrap samples $\xi_{t}^{*}=\left(\varepsilon_{t}^{*}, \eta_{t}^{* \prime}\right)^{\prime}$ by resampling from the centered fitted residual vectors $\left(\tilde{\xi}_{t}\right)=\left(\tilde{\varepsilon}_{t}, \tilde{\eta}_{t}^{\prime}\right)^{\prime}$ where $\left(\tilde{\varepsilon}_{t}\right)$ and $\left(\tilde{\eta}_{t}\right)$ are the fitted residuals from (14) and (15). That is, obtain iid samples $\left(\xi_{t}^{*}\right)$ from the empirical distribution of

$$
\left(\tilde{\xi}_{t}-\frac{1}{n} \sum_{t=1}^{n} \tilde{\xi}_{t}\right)_{t=1}^{n} .
$$

The bootstrap samples $\left(\xi_{t}^{*}\right)$ constructed as such will satisfy $\mathbf{E}^{*} \xi_{t}^{*}=0$ and $\mathbf{E}^{*} \xi_{t}^{*} \xi_{t}^{* \prime}=\tilde{\Sigma}$, where $\tilde{\Sigma}=(1 / n) \sum_{t=1}^{n} \tilde{\xi}_{t} \tilde{\xi}_{t}^{\prime} \cdot 5$

Next, we generate the bootstrap samples for $\left(w_{t}^{*}\right)$ recursively from $\left(\eta_{t}^{*}\right)$ using the fitted autoregression given by

$$
w_{t+r+1}^{*}=\tilde{\Phi}_{1} w_{t+r}^{*}+\cdots+\tilde{\Phi}_{\ell} w_{t+r+1-\ell}^{*}+\eta_{t}^{*}
$$

\footnotetext{
${ }^{5}$ Alternatively, we may resample $\varepsilon_{t}^{*}$ and $\eta_{t}^{*}$ separately from the $\tilde{\varepsilon}_{t}$ and $\tilde{\eta}_{t}$ for $t=1, \ldots, n$. In this case, however, preserving the original correlation structure needs more care. We basically need to pre-whiten $\tilde{\varepsilon}_{t}$ and $\tilde{\eta}_{t}$ before resampling, and then re-color the resamples to recover the correlation structure. More specifically, we first pre-whiten $\tilde{\varepsilon}_{t}$ and $\tilde{\eta}_{t}$ by pre-multiplying $\tilde{\Sigma}^{-1 / 2}$ to $\tilde{\xi}_{t}=\left(\tilde{\varepsilon}_{t}, \tilde{\eta}_{t}^{\prime}\right)^{\prime}$, for $t=1, \ldots, n$. Next, generate $\xi_{t}^{*}=\left(\varepsilon_{t}^{*}, \eta_{t}^{* \prime}\right)^{\prime}$ by resampling from the pre-whitened $\tilde{\varepsilon}_{t}$ and $\tilde{\eta}_{t}$ and subsequently re-coloring them by pre-multiplying $\tilde{\Sigma}^{1 / 2}$ to restore the original dependence structure.
} 
with appropriately chosen $\ell$-initial values of $\left(w_{t}^{*}\right)$, where $\tilde{\Phi}_{k}, 1 \leq k \leq \ell$ are the coefficient estimates from the fitted regression (15). Initialization of $\left(w_{t}^{*}\right)$ is unimportant for our subsequent theoretical development, though it may play an important role in finite samples. ${ }^{6}$ Then we obtain $\left(w_{t+r}^{*}, \ldots, w_{t-q}^{*}\right)$ from the sequence $\left(w_{t}^{*}\right)$, and construct the bootstrap samples $\left(v_{t}^{*}\right)$ as

$$
v_{t}^{*}=\sum_{k=-r}^{q} \tilde{\beta}_{k}^{\prime} w_{t-k}^{*}+\varepsilon_{t}^{*}
$$

using the LS estimates $\tilde{\beta}_{k},-r \leq k \leq q$ from the fitted regression (14). Then we generate $\left(u_{t}^{*}\right)$ recursively from $\left(v_{t}^{*}\right)$ using the fitted autoregression given by

$$
u_{t}^{*}=\tilde{\alpha}_{1} u_{t-1}^{*}+\cdots+\tilde{\alpha}_{p} u_{t-p}^{*}+v_{t}^{*}
$$

with appropriately chosen $p$-initial values of $\left(u_{t}^{*}\right)$, and where $\tilde{\alpha}_{k}, 1 \leq k \leq p$ are the estimates for $\alpha_{k}$ 's from the fitted regression (14).

Finally, we generate $\left(y_{t}^{*}\right)$ from $\left(u_{t}^{*}\right)$ with the null restriction $\alpha=0$ imposed. This is to ensure the nonstationarity of the generated bootstrap samples $\left(y_{t}^{*}\right)$, which is claimed under the null hypothesis, and to make the subsequent bootstrap tests valid. Thus we obtain $\left(y_{t}^{*}\right)$ as

$$
y_{t}^{*}=y_{t-1}^{*}+u_{t}^{*}=y_{0}^{*}+\sum_{k=1}^{t} u_{k}^{*}
$$

which also requires initialization $y_{0}^{*}$. An obvious choice would be to use the initial value $y_{0}$ of $\left(y_{t}\right)$, and generate the bootstrap samples $\left(y_{t}^{*}\right)$ conditional on $y_{0}$. As discussed earlier, the choice of initial value may affect the finite sample performance of the bootstrap; however, the effect of the initial value becomes negligible asymptotically as long as it is stochastically bounded. If the mean or linear time trend is maintained as in (12) or (13) and the unit root test is performed using the demeaned or detrended data, the effect of the initial value $y_{0}^{*}$ of the bootstrap sample would disappear. We may therefore just set $y_{0}^{*}=0$ for the subsequent development of our theory in this section.

To construct the bootstrapped tests, we consider the following bootstrap version of the covariates augmented regression (3), which was used to construct the sample CADF tests $S_{n}$ and $T_{n}$ in the previous section

$$
\triangle y_{t}^{*}=\alpha y_{t-1}^{*}+\sum_{k=1}^{p} \alpha_{k} \Delta y_{t-k}^{*}+\sum_{k=-r}^{q} \beta_{k}^{\prime} w_{t-k}^{*}+\varepsilon_{t}^{*} .
$$

We test for the unit root hypothesis $\alpha=0$ in (20) using the bootstrap versions of the CADF tests, defined in (23) and (24) below, that are constructed analogously as their sample counterparts $S_{n}$ and $T_{n}$ defined in (9) and (10).

\footnotetext{
${ }^{6}$ We may use the first $\ell$-values of $\left(w_{t}\right)$ as the initial values of $\left(w_{t}^{*}\right)$. The bootstrap samples $\left(w_{t}^{*}\right)$ generated as such may not be stationary processes. Alternatively, we may generate a larger number, say $n+M$, of $\left(w_{t}^{*}\right)$ and discard first $M$-values of $\left(w_{t}^{*}\right)$. This will ensure that $\left(w_{t}^{*}\right)$ become more stationary. In this case the initialization becomes unimportant, and we may therefore simply choose zeros for the initial values.
} 
Similarly as before, we denote by $\hat{\alpha}_{n}^{*}$ and $s\left(\hat{\alpha}_{n}^{*}\right)$ respectively the OLS estimator for $\alpha$ and the standard error for $\hat{\alpha}_{n}^{*}$ obtained from the CADF regression (20) based on the bootstrap samples. To define them more explicitly, we let

$$
z_{t}^{*}=\left(\triangle y_{t-1}^{*}, \ldots, \triangle y_{t-p}^{*}, w_{t+r}^{* \prime}, \ldots, w_{t-q}^{* \prime}\right)^{\prime}
$$

and subsequently define

$$
\begin{aligned}
& A_{n}^{*}=\sum_{t=1}^{n} y_{t-1}^{*} \varepsilon_{t}^{*}-\left(\sum_{t=1}^{n} y_{t-1}^{*} z_{t}^{* \prime}\right)\left(\sum_{t=1}^{n} z_{t}^{*} z_{t}^{* \prime}\right)^{-1}\left(\sum_{t=1}^{n} z_{t}^{*} \varepsilon_{t}^{*}\right) \\
& B_{n}^{*}=\sum_{t=1}^{n} y_{t-1}^{* 2}-\left(\sum_{t=1}^{n} y_{t-1}^{*} z_{t}^{* \prime}\right)\left(\sum_{t=1}^{n} z_{t}^{*} z_{t}^{* \prime}\right)^{-1}\left(\sum_{t=1}^{n} z_{t}^{*} y_{t-1}^{*}\right)
\end{aligned}
$$

and the variance of the bootstrap sample $\left(\varepsilon_{t}^{*}\right)$, which is given by

$$
\tilde{\sigma}_{n}^{2}=\frac{1}{n} \sum_{t=1}^{n}\left(\tilde{\varepsilon}_{t}-\bar{\varepsilon}_{n}\right)^{2},
$$

where $\bar{\varepsilon}_{n}=n^{-1} \sum_{t=1}^{n} \tilde{\varepsilon}_{t}$. Then we may write the OLS estimator of $\alpha$ from the bootstrap CADF regression (20) and its estimated variance as

$$
\begin{aligned}
\hat{\alpha}_{n}^{*} & =A_{n}^{*} B_{n}^{*-1} \\
s\left(\hat{\alpha}_{n}^{*}\right)^{2} & =\tilde{\sigma}_{n}^{2} B_{n}^{*-1} .
\end{aligned}
$$

We also define, accordingly as $\hat{\alpha}_{n}(1)$ introduced in (8),

$$
\tilde{\alpha}_{n}(1)=1-\sum_{k=1}^{p} \tilde{\alpha}_{k}
$$

where $\tilde{\alpha}_{k}$ 's are the estimates for $\alpha_{k}$ 's from the fitted regression (14).

Now we consider the statistics

$$
\begin{aligned}
S_{n}^{*} & =\frac{n \hat{\alpha}_{n}^{*}}{\tilde{\alpha}_{n}(1)} \\
T_{n}^{*} & =\frac{\hat{\alpha}_{n}^{*}}{s\left(\hat{\alpha}_{n}^{*}\right)}
\end{aligned}
$$

corresponding to $S_{n}$ and $T_{n}$ introduced in (9) and (10) of the previous section. For the construction of the bootstrap statistics $S_{n}^{*}$ and $T_{n}^{*}$, it is possible to replace $\tilde{\alpha}_{n}(1)$ and $\tilde{\sigma}_{n}^{2}$ with $\hat{\alpha}_{n}^{*}(1)$ and $\hat{\sigma}_{n}^{2 *}$, the bootstrap counterparts to $\hat{\alpha}_{n}(1)$ and $\hat{\sigma}_{n}^{2}$. We can compute $\hat{\alpha}_{n}^{*}(1)$ and $\hat{\sigma}_{n}^{2 *}$ from regression (20) in the same way that their sample counterparts are computed from regression (3). We may indeed show that such replacements do not affect the limiting distributions of the statistics. For the theoretical analysis in the paper, however, we only consider $S_{n}^{*}$ and $T_{n}^{*}$ defined in (23) and (24) for the expositional brevity. 
To implement the bootstrap CADF tests, we repeat the bootstrap sampling for the given original sample and obtain $a_{n}^{*}(\lambda)$ and $b_{n}^{*}(\lambda)$ such that

$$
\mathbf{P}^{*}\left\{S_{n}^{*} \leq a_{n}^{*}(\lambda)\right\}=\mathbf{P}^{*}\left\{T_{n}^{*} \leq b_{n}^{*}(\lambda)\right\}=\lambda
$$

for any prescribed size level $\lambda$. The bootstrap CADF tests reject the null hypothesis of a unit root if

$$
S_{n} \leq a_{n}^{*}(\lambda), T_{n} \leq b_{n}^{*}(\lambda) .
$$

It will now be shown under appropriate conditions that the tests are asymptotically valid, i.e., they have asymptotic size $\lambda$. We do not analyze in the paper the randomness associated with the bootstrap sampling in computing the bootstrap critical values $a_{n}^{*}(\lambda)$ and $b_{n}^{*}(\lambda)$. We simply assume that enough number of bootstrap iterations are carried out to make it negligible. See Andrews and Buchinsky (1999) for a study on the number of bootstrap iterations to achieve the desired level of bootstrap sampling accuracy.

We now introduce the notation $\rightarrow d^{*}$ for bootstrap asymptotics. For a sequence of bootstrapped statistics $\left(Z_{n}^{*}\right)$, we write

$$
Z_{n}^{*} \rightarrow d^{*} Z \text { a.s. }
$$

if the conditional distribution of $\left(Z_{n}^{*}\right)$ weakly converges to that of $Z$ a.s. Here it is assumed that the limiting random variable $Z$ has distribution independent of the original sample realization.

We now present the limit theories for the bootstrap CADF tests $S_{n}^{*}$ and $T^{*}$.

Theorem 3.3 Under the null hypothesis $\alpha=0$, we have as $n \rightarrow \infty$,

$$
\begin{aligned}
S_{n}^{*} \rightarrow_{d}^{*} \sigma_{\varepsilon} \frac{\int_{0}^{1} Q(s) d P(s)}{\int_{0}^{1} Q(s)^{2} d s} \text { a.s. } \\
T_{n}^{*} \rightarrow_{d}^{*} \frac{\int_{0}^{1} Q(s) d P(s)}{\left(\int_{0}^{1} Q(s)^{2} d s\right)^{1 / 2}} \text { a.s. }
\end{aligned}
$$

under Assumptions 2.1 and 2.2, where $Q(s)$ and $P(s)$ are defined in Theorem 2.3.

Theorem 3.3 shows that the bootstrap statistics $S_{n}^{*}$ and $T_{n}^{*}$ have the same null limiting distributions as the corresponding sample statistics $S_{n}$ and $T_{n}$. It implies, in particular, that the bootstrap CADF tests are asymptotically valid.

To discuss the asymptotic validity of the tests using bootstrap critical values, denote by $S$ and $T$ the weak limits of $S_{n}$ and $T_{n}$ respectively, and define $a(\lambda)$ and $b(\lambda)$ to be the asymptotic critical values of the size $\lambda$ tests based on $S_{n}$ and $T_{n}$, i.e.,

$$
\mathbf{P}\{S \leq a(\lambda)\}=\mathbf{P}\{T \leq b(\lambda)\}=\lambda .
$$


Since the distributions of $S$ and $T$ are absolutely continuous with respect to Lebesgue measure, we have from Theorem 3.3

$$
\mathbf{P}^{*}\left\{S_{n}^{*} \leq a(\lambda)\right\}, \mathbf{P}^{*}\left\{T_{n}^{*} \leq b(\lambda)\right\} \rightarrow \lambda \text { a.s. }
$$

under Assumptions 2.1 and 2.2, and the results in (26) imply

$$
\left(a_{n}^{*}(\lambda), b_{n}^{*}(\lambda)\right) \rightarrow(a(\lambda), b(\lambda)) \text { a.s. },
$$

where $a_{n}^{*}(\lambda)$ and $b_{n}^{*}(\lambda)$ are the size $\lambda$ bootstrap critical values defined in (25). Consequently, we have under Assumptions 2.1 and 2.2

$$
\mathbf{P}\left\{S_{n} \leq a_{n}^{*}(\lambda)\right\}, \mathbf{P}\left\{T_{n} \leq b_{n}^{*}(\lambda)\right\} \rightarrow \lambda
$$

as $n \rightarrow \infty$, which proves that the bootstrap CADF tests have size $\lambda$ asymptotically.

Our bootstrap theory here easily extends to the tests for a unit root in models with deterministic trends, such as those introduced in (12) or (13). It is straightforward to establish the bootstrap consistency for the CADF tests applied to the demeaned and detrended time series, using the results obtained in this section. The bootstrap CADF tests are therefore valid and applicable also for the models with deterministic trends.

\section{Simulations}

We perform a set of simulations to investigate the finite sample performances of the bootstrap CADF $t$-test. For the simulations, we consider $\left(y_{t}\right)$ given by the unit root model (1) with the error $\left(u_{t}\right)$ generated by

$$
u_{t}=\alpha_{1} u_{t-1}+v_{t}
$$

where the error term $\left(v_{t}\right)$ is given by

$$
v_{t}=\beta w_{t}+\varepsilon_{t}
$$

In our simulation study, the covariate $\left(w_{t}\right)$ is assumed to follow an $\operatorname{AR}(1)$ process

$$
w_{t+1}=\phi w_{t}+\eta_{t}
$$

The innovations $\xi_{t}=\left(\varepsilon_{t}, \eta_{t}\right)^{\prime}$ are i.i.d. $\mathbf{N}(0, \Sigma)$, where

$$
\Sigma=\left(\begin{array}{cc}
1 & \sigma_{\varepsilon \eta} \\
\sigma_{\eta \varepsilon} & 1
\end{array}\right) .
$$

Under this setup, we have the following covariate augmented ADF regression:

$$
\Delta y_{t}=\alpha y_{t-1}+\alpha_{1} \Delta y_{t-1}+\beta w_{t}+\varepsilon_{t} .
$$

The correlation between the innovation $v_{t}=\beta w_{t}+\varepsilon_{t}$ and the covariate $w_{t}$ depends on two parameter values, the coefficient $\beta$ on the covariate and the AR coefficient $\phi$ of the covariate, 
as can be seen clearly from the data generating process (DGP). Consequently, the relative merit of constructing a unit root test from the covariate augmented regression depends on the parameters $\beta$ and $\phi$. We control the degree of correlation between the error $v_{t}$ and the covariate $w_{t}$ through these parameters. The values of the parameters $\beta$ and $\phi$ are allowed to vary among $\{-0.8,-0.5,0.5,0.8\}$. Parameter $\alpha_{1}$ on the lagged difference term does not affect the aforementioned correlation and, hence, we set $\alpha_{1}=0$ throughout the simulations for convenience. The contemporaneous covariance, $\sigma_{\varepsilon \eta}$, is set at 0.5 .

For the test of the unit root hypothesis, we set $\alpha=0$ and investigate finite sample sizes in relation to corresponding nominal test sizes. For finite sample powers, we consider the cases, $\alpha=-0.05$, and -0.10 . The finite sample performances of the bootstrap CADF test are compared with those of the sample CADF test computed from the regression (27) and also with those of the ADF test based on the usual ADF regression. The usual ADF regression does not include the covariate $w_{t}$ as a regressor and thus the regression error effectively becomes $v_{t}=\beta w_{t}+\varepsilon_{t}$ with the DGP considered in this simulation setup. The effective error $v_{t}$ is obviously serially correlated due to serial correlation in $w_{t}$. This invalidates the use of the conventional ADF test which uses the critical values from the Dickey-Fuller distribution. To make more meaningful comparisons with the results of the ADF test, we may increase the number of lagged differences to whiten the error $v_{t}$. In this simulation experiment we set the lag order for the ADF test at $3 .^{7}$

The regression equation for the CADF test contains no $\mathrm{AR}$ lag terms and includes only the current value of the covariate in each regression. All regressions include a fitted intercept. The regression equation for covariate (15) is estimated using AR(1) model. To implement the CADF test, asymptotic critical values, corresponding to each sample estimate of $\rho^{2}$, are taken from Table 1 in Hansen (1995). ${ }^{8}$ Sample sizes of $n=50,100$, and 250 are examined for $1 \%, 5 \%$, and $10 \%$ nominal size tests; we report the results for $n=100$ only, since those for $n=50$, and 250 are qualitatively similar. Each replication discards the first 100 observations to eliminate start-up effects. The reported results are based on 5,000 simulation iterations with the bootstrap critical values computed from 3,000 bootstrap repetitions. The finite sample sizes and powers for the ADF, the CADF, and the bootstrap CADF tests are reported, respectively, in Tables 1 and 2.

As can be seen clearly from Table 1, the bootstrap CADF test significantly improves the finite sample size performances of the sample CADF test, especially when the covariates are highly correlated with the error. More precisely, when the parameter $\beta$ is large in absolute value and the parameter $\phi$ is large with positive value, the size distortions of the CADF test are quite noticeable. The downward size distortions of the CADF test for $(\beta, \phi)=(-0.8,0.8)$ and $(-0.5,0.8)$ are very serious while the overrejections for $(\beta, \phi)=(0.8,0.8)$ and $(0.5,0.8)$ are not negligible. This tendency becomes more severe as the contemporaneous covariance, $\sigma_{\varepsilon \eta}$, gets large in absolute value. For example, when $\sigma_{\varepsilon \eta}=0.8$, actual $10 \%$ size of the CADF test for $(\beta, \phi)=(0.8,0.8)$ goes up to $16 \%$ and in the case of $(\beta, \phi)=(-0.8,0.8)$ it

\footnotetext{
${ }^{7}$ The ADF test with lag order smaller than 3 tends to overreject the null hypothesis. The lag order 3 seems to have the best overall size and power performances and thus is chosen for our simulations.

${ }^{8}$ Sample estimates of $\rho^{2}$ are calculated using the Parzen kernel and Andrews' (1991) automatic bandwidth estimator.
} 
goes down to as low as $1 \% .{ }^{9}$ In the case of the bootstrap CADF test, however, we do not observe such size distortions. The bootstrap CADF test performs generally very well for different choices of parameters. The size distortions of the ADF test are little or mild.

The significant improvement in the finite sample sizes that the bootstrap CADF test offers does not come at the expense of finite sample powers. Indeed, the results in Table $2 \mathrm{~A}$ and $2 \mathrm{~B}$ show that the bootstrap CADF test also offers drastic power gains over the conventional $\mathrm{ADF}$ test for all the $1 \%, 5 \%$, and $10 \%$ tests. In particular, the discriminatory powers of the bootstrap CADF test are noticeably much higher than those of the ADF test for the cases when the parameter $\beta$ has positive values. For $\beta=0.8$, the powers of the bootstrap CADF test for $\alpha=-0.10$ are more than three times as large as those of the ADF test. The discrepancies become four times as large when $\alpha=-0.05$. The bootstrap CADF test performs much better than the ADF test for low values of $\hat{\rho}^{2}$ when $\beta$ is negative. Note that the powers of the bootstrap CADF test are comparable to those of the CADF test in most cases, and even better in some cases.

\section{Empirical Applications}

We next apply our testing procedures to a set of U.S. macroeconomic aggregates and OECD financial time series. A number of econometric studies have found that standard tests for a unit root, such as the ADF (Dickey and Fuller, 1979, 1981) and the Phillips and Perron (1988) tests, have low power against stationary alternatives in the relatively small samples we consider in this section (See, Dejong, Nankervis, Savin, and Whiteman 1992a, 1992b, among others). This is especially true when a series under investigation is a near-integrated process. Since the low power of the univariate unit root tests is the primary problem, it is important to investigate whether or not the null hypothesis of a unit root is rejected by the more powerful covariate tests. Moreover, various univariate unit root tests provide mixed results for a given time series when they do not consistently reject or accept the null hypothesis. In this situation making a definitive conclusion about the (non)stationarity of the time series may be problematic. Hence, the use of more powerful tests may point to sharper conclusions about the stationarity property of the particular time series.

We consider two data sets. The first is the Nelson-Plosser data set extended by Schotman and Van Dijk (1991) to end in 1988. The second is annual CPI-based real exchange rates ${ }^{10}$ for 14 OECD countries from 1951 to 1998. The real exchange rate, $r_{i t}$, for the $i$-th country is computed using the U.S. dollar as numeraire currency. Real exchange rates are analyzed for Australia, Austria, Belgium, Canada, France, Finland, Germany, Italy, Japan, Luxemburg, Netherlands, Norway, Spain, and the United Kingdom.

The testing strategy is as follows. Note first that the order of autoregression has important effects on the size and power performances of the tests (see $\mathrm{Ng}$ and Perron 1995, and Schwert 1989, among others). Secondly, note also that a deterministic rule relating lag length $p$ to sample size $n$ is inferior to a data-dependent rule that takes sample information

\footnotetext{
${ }^{9}$ The result for $\sigma_{\varepsilon \eta}=0.8$ is not reported in the paper. It is available upon request from the authors.

${ }^{10}$ The real exchange rate is calculated as $r_{i t}=\log \left(e_{i t} p_{* t} / p_{i t}\right)$, where $e_{i t}, p_{* t}$, and $p_{i t}$ denote respectively nominal spot exchange rate for the $i$-th country, the CPI for the U.S., and the CPI for the $i$-th country.
} 
into account ( $\mathrm{Ng}$ and Perron 1995). Based on these findings, we first use the ADF test with different lag order selection criteria such as AIC, BIC, and STC (the sequential test criterion $^{11}$ ), and the Phillips and Perron (PP) tests, $Z_{t}$ and $Z_{\alpha}$, for different choices of truncation lag parameter. If all of these tests consistently reject or accept the null hypothesis of a unit root for a particular time series, we will use that variable as a possible candidate for a covariate. If the variable to be used as a covariate is stationary, then we will use the level of it, and if the variable is nonstationary, then we will take a first difference of it. Thus, only stationary covariates will be utilized in our multivariate tests.

To the time series whose test results are undetermined, we apply the CADF and the bootstrap CADF tests with covariates selected by the above 'pre-tests' which are now known to be either $\mathrm{I}(0)$ or $\mathrm{I}(1)$. Among the candidates for covariates we choose the one which gives us the smallest $\hat{\rho}^{2}$ since this covariate provides the most powerful test, as shown in Section 4. Unless otherwise stated, all regressions include a constant and a time trend. For the covariate tests, we do not use future covariate values, i.e., we let $r=0$ in all the regressions. The AIC lag order selection rule is used as a lag selection criterion for the CADF test and for the AR estimation of covariates. For the CADF test, asymptotic critical values, corresponding to each sample estimate of $\rho^{2}$, are taken from Table 1 in Hansen (1995). For the bootstrap test we use critical values computed from 5,000 bootstrap iterations.

\subsection{The Extended Nelson-Plosser Data Series}

The Nelson and Plosser data set is one of the most widely analyzed macroeconomic aggregate time series data sets. Nelson and Plosser (1982) studied the time series properties of 14 series and found that all of them, with the exception of the unemployment rate series, were characterized by stochastic nonstationarity. In our empirical application we use the extended Nelson-Plosser data set of Schotman and Van Dijk (1991). All variables are measured in logarithms. The estimated period is 1930-1988 in consideration of a structural break in 1929 coinciding with the onset of the Great Depression.

We perform the ADF and the PP tests for the 14 time series whose non-stationarity have been questioned. Table 3 presents the results. With the ADF test we reject the null hypothesis for 12 series at least at the $10 \%$ significance level. With the PP test, the unit root hypothesis can be rejected for 9 series at least at the $10 \%$ significance level. Both tests fail to reject the unit root hypothesis for real wages. The null hypothesis is rejected using both tests for 4 series: real GNP, real per capita GNP, employment, and unemployment rate $^{12}$. The standard tests clearly do not give definitive conclusions for 9 out of 14 time series (nominal GNP, industrial production, GNP deflator, consumer prices, wages, money stock, velocity, interest rate, and common stock prices). Panel (c) of Table 3 contains

\footnotetext{
${ }^{11}$ The value of lag length $p$ chosen is determined by a test on the significance of the estimated coefficients $\hat{\alpha}_{i}$. We actually used a fairly liberal procedure choosing a value of $p$ equal to $p^{*}$ if the $t$ statistic on $\hat{\alpha}_{i}$ was greater than 1.60 in absolute value and the $t$ statistic on $\hat{\alpha}_{i}$ for $l>p^{*}$ was less than 1.60 (with a maximum value for $p$ of 8 for the Nelson-Plosser series, and 6 for real exchange rates). This liberal procedure is justified in the sense that including too many extra regressors of lagged first-differences does not affect the size of the test but only decreases its power. Including too few lags may have a substantial effect on the size of the test (Perron 1989, and Ng and Perron 1995).

${ }^{12}$ In the case of the unemployment rate series, the result is obtained without a time trend in the regression.
} 
the test results of the remaining 5 series for which the null hypothesis can be consistently accepted or rejected. ${ }^{13}$ We will use these 5 series as covariates for the CADF test. Note that $\mathrm{I}(1)$ variables will be first-differenced before they are used as covariates. ${ }^{14}$

To the 9 time series whose results are undetermined, we apply the covariate tests using as covariates the series consistently found to be either $\mathrm{I}(0)$ or $\mathrm{I}(1)$ by the above pre-tests. The results are given in Panel (a) of Table 3. For all cases the values of $\hat{\rho}^{2}$ are lower than 0.1 ; thus we should expect, based on our simulation results, more powerful test results with the CADF and the bootstrap CADF tests than with the ADF test. With these new tests we can reject the null hypothesis of a unit root for 8 time series. The only exception is for the series on industrial production. Non-stationarity of the GNP deflator, wages, velocity, interest rate, and common stock prices series are strongly rejected at the $1 \%$ significance level. These results are fairly robust to the choice of maximum lag length in the cases of nominal GNP, industrial production, velocity, and interest rate, while for the other series the tests tend to reject the null hypothesis for higher orders of lag length. The results show that with the more powerful covariate tests there is much less uncertainty about the potential stationarity of many commonly studied U.S. macroeconomic aggregates.

We can compare our findings with those of Perron (1989). For the post-1929 period, he could not reject the null hypothesis of a unit root for interest rate. With the CADF and the bootstrap CADF tests, however, we can reject the unit root hypothesis in the interest rate series at the $1 \%$ significance level. We cannot, however, reject the null hypothesis for real wages and industrial production. To check the possibility that this difference may come from the effect of the 1973 oil shock, we analyze the two series for the period of 1930-1972. Results are shown in Panel (b) of Table 3. The ADF and the PP tests give us mixed results, while the $\mathrm{CADF}$ and the bootstrap CADF tests strongly reject the null hypothesis at the $1 \%$ significance level. Therefore, the CADF and the bootstrap CADF tests reject the nonstationarity of all the series in the Nelson-Plosser data set for the post-1929 samples.

Given that the unit root hypothesis can be rejected for all the series, we can assess the significance of the other coefficients since the asymptotic distributions of their $t$-statistics are standard normal. In all cases except consumer prices, interest rate, and common stock prices, the time trend coefficients are significant at least at the $5 \%$ level. These results suggest that the underlying processes of these series are characterized mainly by stationary fluctuations around a deterministic trend function for the sample period. The consumer prices, interest rate and common stock prices series are analyzed without a time trend and the results indicate that these series are stationary around a constant.

\footnotetext{
${ }^{13}$ We varied the maximum lag length, $q_{\max }$, from 2 to 8 for the ADF test, and similarly from 2 to 8 for the choice of truncation lag parameter for the PP test. The results seem to be robust to the choice of maximum lag lengths.

${ }^{14}$ Stock and Watson (1999) note that current theoretical literatures in macroeconomics provide neither intuition nor guidance on which covariates are candidates for our CADF and bootstrap CADF tests other than on the basis of stationarity.
} 


\subsection{Real Exchange Rates}

Another situation in which the covariate tests are especially useful is with certain types of panel data in which cross-sectional correlations between time series are present. If this information can be properly modeled then it can provide an efficiency gain over univariate methods as pointed out by Hansen (1995). To properly exploit these potential efficiency (and power) gains, we analyze annual CPI-based real exchange rates for 14 OECD countries for the period of 1951-1998, the period including the Bretton Woods system and flexible exchange rate regime. We have a bit more intuition here as to which covariates may be more important since covariance with close trading partners has a geographical as well as an economic rationale.

Movements in real exchange rates are thought to be driven primarily by deviations from purchasing power parity (PPP). Models of exchange rate determination are built on the assumption that the PPP hypothesis holds. There are, however, conflicting empirical evidences. Recent studies by MacDonald (1996), Frankel and Rose (1996), Oh (1996), Papell (1997), and O'Connell (1998), among others, suggest that the issue is not completely settled. In particular, when considering data for the recent flexible rate experience (1973present), many researchers have been unable to reject the null hypothesis of a unit root (see, e.g., Mark 1990, and Edison and Pauls 1993). One response to this non-rejection might be that the tests do not encompass a sufficiently long time-span to capture the mean reversion necessary to reject the null hypothesis. It is of interest, therefore, if real exchange rates are mean reverting for longer spans of time than the period of recent float regime, using low frequency data. One advantage of using relatively low frequency annual or quarterly data is that it can possibly increase the power of statistical tests for random walk behavior (Shiller and Perron 1985).

As pretests, the ADF and the PP tests are performed for the 14 real exchange rate series. With the ADF test, we can reject the unit root hypothesis for 9 countries, while the null hypothesis is rejected for 12 countries with the PP test. We cannot reject the null hypothesis with both tests for the Netherlands and Norway, and both tests consistently reject the null hypothesis of a unit root for 6 countries. Table 4 shows the result. We therefore have 6 remaining countries whose stationarity properties are undetermined (Australia, Austria, Canada, Japan, Belgium, and Germany).

The results of the covariate tests for the 6 countries are in Panel (a) of Table 4. For the chosen covariates, the estimated $\hat{\rho}^{2}$ are quite low, ranging between 0.001 and 0.042 . This indicates that the powers of the CADF and the bootstrap CADF tests should be considerably higher than those of the ADF test (and possibly higher than those of the PP test). Results based on the CADF and the bootstrap CADF tests now indicate that we can reject the null hypothesis of a unit root for all the countries, although the evidence for Belgium and Germany is less compelling than for Australia, Austria, Canada, Japan, and the U.K. for which the null hypothesis of a unit root is strongly rejected at the $1 \%$ significance level ( $5 \%$ level in the case of Australia). ${ }^{15}$

\footnotetext{
${ }^{15}$ For these two countries, the CADF test cannot reject the null hypothesis. However, the critical values of the $10 \%$ test are -1.753 and -1.952 , respectively, and their test statistics, $t=-1.560$ and -1.811 , respectively, are very close to the critical values. Thus the evidence for these two countries is marginal.
} 
Our results based on the more powerful covariate tests are at variance with those based on standard univariate unit root tests. Frankel (1985) tested for a unit root in the real exchange rate of the United Kingdom using the Dickey-Fuller test on annual data and was unable to reject the random walk hypothesis when the sample period was limited to the postwar era (1945-1984). Whitt (1992) tested for a unit root in the real exchange rates of the U.K., Japan, Australia, and several other countries using the Dickey-Fuller test on annual data post World War II and was unable to reject the null hypothesis of a unit root for these countries. Flynn and Boucher (1993), using monthly data for the similar period, conducted tests for the PPP hypothesis and concluded that PPP does not hold for Canada and Japan. Now, with an expanded data set (through 1998) and more powerful tests, we can decisively reject the null hypothesis of a unit root for Australia, Canada, Japan, and the U.K.. These findings are supportive of the idea of Aizenman (1984) on the PPP hypothesis that "the doctrine of PPP should hold better between neighboring countries, and between countries with larger potential trade, because of the lower transaction cost of trade in goods between such countries." 16

\section{Conclusion}

In this paper, we consider the bootstrap procedure for the covariates augmented DickeyFuller (CADF) unit root test which substantially improves the power of univariate unit root tests. Hansen (1995) originally proposed the CADF test and suggested a two-step procedure to overcome the nuisance parameter dependency problem. Here, we propose bootstrapping the CADF test in order to directly deal with the nuisance parameter dependency and base inferences on the bootstrapped critical values. We also establish the bootstrap consistency of the CADF test and show that the bootstrap CADF test is asymptotically valid.

The finite sample performances of the bootstrap CADF test are investigated and compared with those of the CADF test and the usual ADF test through simulations. The bootstrap CADF test significantly improves the finite sample size performances of the CADF test, especially when the covariates are highly correlated with the error. Indeed, the bootstrap CADF test offers drastic power gains over the conventional ADF test. As an illustration, we apply the tests to the 14 macroeconomic time series in the extended Nelson-Plosser data set for the post-1929 samples as well as postwar annual CPI-based real exchange rates for 14 OECD countries. In contrast to the results of previous studies using the univariate unit root tests, our empirical results show that the null hypothesis of a unit root is rejected for all the series in the Nelson-Plosser data set and for most of the countries in the real exchange rates series.

\section{Appendix}

Lemma A.1 Under Assumption 2.1, we have as $n \rightarrow \infty$

\footnotetext{
${ }^{16}$ In particular, Canada and Japan are ranked as the U.S.'s top two trading partners.
} 
(a) $\frac{1}{\sqrt{n}} \sum_{t=1}^{[n s]} w_{t} \rightarrow_{d} \Psi(1) B_{\eta}(s)$

(b) $\frac{1}{\sqrt{n}} \sum_{t=1}^{[n s]} u_{t} \rightarrow_{d} \pi(1)\left(\beta(1)^{\prime} \Psi(1) B_{\eta}(s)+B_{\varepsilon}(s)\right)$

where $\Psi(1)=\Phi(1)^{-1}$ and $\pi(1)=1 / \alpha(1)$.

Proof of Lemma A.1 To establish the stated results, we use the Beveridge-Nelson (BN) representations for the finite order lag polynomials $\alpha(L), \beta(L)$ and $\Phi(L)$ defined in (2) and (4) and the limit theory from the invariance principle given in (5).

Part (a) We begin by deriving the BN representation for $\left(w_{t}\right)$ from (4). Let $\Phi(1)=$ $I_{m}-\sum_{k=1}^{\ell} \Phi_{k}$. Then we may easily get

$$
\Phi(1) w_{t}=\eta_{t-r-1}+\sum_{k=1}^{\ell} \sum_{j=k}^{\ell} \Phi_{j}\left(w_{t-k}-w_{t-k+1}\right)
$$

or

$$
w_{t}=\Psi(1) \eta_{t-r-1}+\left(\bar{w}_{t-1}-\bar{w}_{t}\right),
$$

where $\Psi(1)=\Phi(1)^{-1}$ and $\bar{w}_{t}=\sum_{k=1}^{\ell} \bar{\Phi}_{k} w_{t-k+1}$, with $\bar{\Phi}_{k}=\Psi(1) \sum_{j=k}^{\ell} \Phi_{j}$. Under our condition in Assumption 2.1, $\left\{\bar{w}_{t}\right\}$ is well defined both in a.s. and $L^{s}$ sense [see Brockwell and Davis (1991, Proposition 3.1.1)]. Then we have

$$
\sum_{k=1}^{t} w_{k}=\Psi(1) \sum_{k=1}^{t} \eta_{k-r-1}+\left(\bar{w}_{0}-\bar{w}_{t}\right)
$$

Note that $\left(\bar{w}_{t}\right)$ is stochastically of smaller order of magnitude than the sum $\sum_{k=1}^{t} \eta_{k}$, and hence will become negligible in the limit. Then it follows directly from (5)

$$
\frac{1}{\sqrt{n}} \sum_{t=1}^{[n s]} w_{t}=\Psi(1) \frac{1}{\sqrt{n}} \sum_{t=1}^{[n s]} \eta_{t-r-1}+\frac{1}{\sqrt{n}}\left(\bar{w}_{0}-\bar{w}_{[n s]}\right) \rightarrow_{d} \Psi(1) B_{\eta}(s)
$$

for $s \in[0,1]$, giving the stated result in part (a).

Part (b) Let $\alpha(1)=1-\sum_{k=1}^{p} \alpha_{k}$. Similarly, we derive the BN representation for $u_{t}$ from (2) as follows

$$
\begin{aligned}
u_{t} & =\frac{1}{\alpha(1)}\left(\beta(L)^{\prime} w_{t}+\varepsilon_{t}\right)+\sum_{k=1}^{p} \frac{\sum_{j=k}^{p} \alpha_{j}}{\alpha(1)}\left(u_{t-k}-u_{t-k+1}\right) \\
& =\pi(1)\left(\beta(L)^{\prime} w_{t}+\varepsilon_{t}\right)+\left(\bar{u}_{t-1}-\bar{u}_{t}\right)
\end{aligned}
$$

where $\pi(1)=1 / \alpha(1)$ and $\bar{u}_{t}=\sum_{k=1}^{p} \bar{\alpha}_{k} u_{t-k+1}$, with $\bar{\alpha}_{k}=\pi(1) \sum_{j=k}^{p} \alpha_{j}$. The process $\left\{\bar{u}_{t}\right\}$ is also well defined both in a.s. and $L^{r}$ sense. We may also obtain the $\mathrm{BN}$ representation for $\beta(L)^{\prime} w_{t}$ as follows

$$
\begin{aligned}
\beta(L)^{\prime} w_{t} & =\beta(1)^{\prime} w_{t}+\sum_{k=0}^{q-1} \sum_{j=k+1}^{q} \beta_{j}^{\prime}\left(w_{t-k-1}-w_{t-k}\right)+\sum_{k=0}^{r-1} \sum_{j=k+1}^{r} \beta_{-j}^{\prime}\left(w_{t+k+1}-w_{t+k}\right) \\
& =\beta(1)^{\prime} w_{t}+\left(\bar{w}_{t-1}^{+}-\bar{w}_{t}^{+}\right)+\left(\bar{w}_{t}^{-}-\bar{w}_{t-1}^{-}\right)
\end{aligned}
$$


where $\bar{w}_{t}^{+}=\sum_{k=0}^{q-1} \bar{\beta}_{k}^{+\prime} w_{t-k}$ and $\bar{w}_{t}^{-}=\sum_{k=0}^{r-1} \bar{\beta}_{k}^{-\prime} w_{t+k+1}$, with $\bar{\beta}_{k}^{+}=\sum_{j=k+1}^{q} \beta_{j}$ and $\bar{\beta}_{k}^{-}=$ $\sum_{j=k+1}^{r} \beta_{-j}$. The $\left\{\bar{w}_{t}^{+}\right\}$and $\left\{\bar{w}_{t}^{-}\right\}$are well defined both in a.s. and $L^{r}$ sense.

Then it follows that

$$
u_{t}=\pi(1)\left(\beta(1)^{\prime} w_{t}+\varepsilon_{t}\right)+\pi(1)\left(\left(\bar{w}_{t-1}^{+}-\bar{w}_{t}^{+}\right)+\left(\bar{w}_{t}^{-}-\bar{w}_{t-1}^{-}\right)\right)+\left(\bar{u}_{t-1}-\bar{u}_{t}\right)
$$

and

$$
\frac{1}{\sqrt{n}} \sum_{t=1}^{[n s]} u_{t}=\pi(1)\left(\beta(1)^{\prime} \frac{1}{\sqrt{n}} \sum_{t=1}^{[n s]} w_{t}+\frac{1}{\sqrt{n}} \sum_{t=1}^{[n s]} \varepsilon_{t}\right)+o_{p}(1)
$$

since

$$
\begin{aligned}
\frac{1}{\sqrt{n}} \sum_{t=1}^{[n s]}\left(\bar{u}_{t-1}-\bar{u}_{t}\right) & =\frac{1}{\sqrt{n}}\left(\bar{u}_{0}-\bar{u}_{[n s]}\right)=o_{p}(1) \\
\frac{1}{\sqrt{n}} \sum_{t=1}^{[n s]}\left(\left(\bar{w}_{t-1}^{+}-\bar{w}_{t}^{+}\right)+\left(\bar{w}_{t}^{-}-\bar{w}_{t-1}^{-}\right)\right) & =\frac{1}{\sqrt{n}}\left(\bar{w}_{0}^{+}-\bar{w}_{[n s]}^{+}\right)+\frac{1}{\sqrt{n}}\left(\bar{w}_{[n s]}^{-}-\bar{w}_{0}^{-}\right)=o_{p}(1) .
\end{aligned}
$$

Now the stated result is immediate from the invariance principle given in (5) and the result in part (a).

Lemma A.2 Under Assumption 2.1, we have

(a) $n^{-2} \sum_{t=1}^{n} y_{t-1}^{2} \rightarrow_{d} \pi(1)^{2} \int_{0}^{1} Q(s)^{2} d s$

(b) $n^{-1} \sum_{t=1}^{n} y_{t-1} \varepsilon_{t} \rightarrow_{d} \sigma_{\varepsilon} \pi(1) \int_{0}^{1} Q(s) d P(s)$

as $n \rightarrow \infty$, where $Q$ and $P$ are defined in Theorem 2.3.

Proof of Lemma A.2 When $\alpha=0$, we have

$$
y_{t}=y_{t-1}+u_{t}=\sum_{k=1}^{t} u_{k}
$$

since $y_{0}=0$. Then it follows from Lemma A.1 (b) that

$$
\frac{1}{\sqrt{n}} y_{[n s]}=\frac{1}{\sqrt{n}} \sum_{t=1}^{[n s]} u_{t} \rightarrow_{d} \pi(1) Q(s)
$$

using the notation introduced in Theorem 2.3. The stated result in part (a) now follows immediately from (30) and the continuous mapping theorem. For part (b), we also have from (30) that

$$
\begin{aligned}
\frac{1}{n} \sum_{t=1}^{n} y_{t-1} \varepsilon_{t} & =\frac{1}{\sqrt{n}} \sum_{t=1}^{n} \frac{y_{t-1}}{\sqrt{n}} \varepsilon_{t} \\
& \rightarrow{ }_{d} \sigma_{\varepsilon} \pi(1) \int_{0}^{1} Q(s) d P(s)
\end{aligned}
$$


as required, where $P$ is the normalized Brownian motion of $B_{\varepsilon}$, i.e., $P=B_{\varepsilon} / \sigma_{\varepsilon}$.

Proof of Theorem 2.3 We have from Lemma 2.1 of Park and Phillips (1989) that

$$
\sum_{t=1}^{n} z_{t} z_{t}^{\prime}=O_{p}(n), \quad \sum_{t=1}^{n} z_{t} \varepsilon_{t}=O_{p}\left(n^{1 / 2}\right), \quad \text { and } \quad \sum_{t=1}^{n} y_{t-1} z_{t}^{\prime}=O_{p}(n) .
$$

Then it follows that

$$
\begin{aligned}
&\left|\left(\sum_{t=1}^{n} y_{t-1} z_{t}^{\prime}\right)\left(\sum_{t=1}^{n} z_{t} z_{t}^{\prime}\right)^{-1}\left(\sum_{t=1}^{n} z_{t} \varepsilon_{t}\right)\right| \leq\left|\sum_{t=1}^{n} y_{t-1} z_{t}^{\prime}\right|\left|\left(\sum_{t=1}^{n} z_{t} z_{t}^{\prime}\right)^{-1}\right|\left|\sum_{t=1}^{n} z_{t} \varepsilon_{t}\right|=O_{p}\left(n^{1 / 2}\right) \\
&\left|\left(\sum_{t=1}^{n} y_{t-1} z_{t}^{\prime}\right)\left(\sum_{t=1}^{n} z_{t} z_{t}^{\prime}\right)^{-1}\left(\sum_{t=1}^{n} z_{t} y_{t-1}\right)\right| \leq\left|\sum_{t=1}^{n} y_{t-1} z_{t}^{\prime}\right|\left|\left(\sum_{t=1}^{n} z_{t} z_{t}^{\prime}\right)^{-1}\right|\left|\sum_{t=1}^{n} z_{t} y_{t-1}\right|=O_{p}(n) \\
&\left|\left(\sum_{t=1}^{n} \varepsilon_{t} z_{t}^{\prime}\right)\left(\sum_{t=1}^{n} z_{t} z_{t}^{\prime}\right)^{-1}\left(\sum_{t=1}^{n} z_{t} \varepsilon_{t}\right)\right| \leq\left|\sum_{t=1}^{n} \varepsilon_{t} z_{t}^{\prime}\right|||\left(\sum_{t=1}^{n} z_{t} z_{t}^{\prime}\right)^{-1}|| \sum_{t=1}^{n} z_{t} \varepsilon_{t} \mid=o_{p}(n) .
\end{aligned}
$$

Hence,

$$
\begin{aligned}
n^{-1} A_{n} & =n^{-1} \sum_{t=1}^{n} y_{t-1} \varepsilon_{t}+o_{p}(1) \\
n^{-2} B_{n} & =n^{-2} \sum_{t=1}^{n} y_{t-1}^{2}+o_{p}(1) \\
n^{-1} C_{n} & =n^{-1} \sum_{t=1}^{n} \varepsilon_{t}^{2}+o_{p}(1) .
\end{aligned}
$$

Under the null, $\alpha=0$ and we have from (9) that

$$
S_{n}=\frac{n B_{n}^{-1} A_{n}}{\hat{\alpha}_{n}(1)}=\frac{1}{\hat{\alpha}_{n}(1)}\left(\frac{n^{-1} \sum_{t=1}^{n} y_{t-1} \varepsilon_{t}}{n^{-2} \sum_{t=1}^{n} y_{t-1}^{2}}\right)+o_{p}(1) \rightarrow_{d} \sigma_{\varepsilon} \frac{\int_{0}^{1} Q(s) d P(s)}{\int_{0}^{1} Q(s)^{2} d s}
$$

as required, due to Lemma A.2. Similarly, the stated limit distribution of $T_{n}$ follows directly from (10) and Lemma A.2 as

$$
T_{n}=\frac{1}{\hat{\sigma}_{n}}\left(\frac{A_{n}}{B_{n}^{1 / 2}}\right)=\frac{1}{\hat{\sigma}_{n}}\left(\frac{n^{-1} \sum_{t=1}^{n} y_{t-1} \varepsilon_{t}}{\left(n^{-2} \sum_{t=1}^{n} y_{t-1}^{2}\right)^{1 / 2}}\right)+o_{p}(1) \rightarrow_{d} \frac{\int_{0}^{1} Q(s) d P(s)}{\left(\int_{0}^{1} Q(s)^{2} d s\right)^{1 / 2}}
$$

since $\hat{\sigma}_{n}^{2} \rightarrow p \sigma_{\varepsilon}^{2}$ and $\pi(1)=1 / \alpha(1)$. 
For the derivation of the limit distributions for the bootstrap CADF tests $S_{n}^{*}$ and $T_{n}^{*}$, we rely on the invariance principles for the bootstrapped samples, which are analogous to those derived for the original samples in Lemma A.1. We will use the symbol $o_{p}^{*}(1)$ to signify the bootstrap convergence in probability. For a sequence of bootstrapped random variables $Z_{n}^{*}$, for instance, $Z_{n}^{*}=o_{p}^{*}(1)$ a.s. and in $\mathbf{P}$ imply respectively that

$$
\mathbf{P}^{*}\left\{\left|Z_{n}^{*}\right|>\delta\right\} \rightarrow 0 \text { a.s. or in } \mathbf{P}
$$

for any $\delta>0$. Similarly, we will use the symbol $O_{p}^{*}(1)$ to denote the bootstrap version of the boundedness in probability. Needless to say, the definitions of $o_{p}^{*}(1)$ and $O_{p}^{*}(1)$ naturally extend to $o_{p}^{*}\left(c_{n}\right)$ and $O_{p}^{*}\left(c_{n}\right)$ for some nonconstant numerical sequence $\left(c_{n}\right)$. Many of well known results for $o_{p}$ and $O_{p}$ extend to $o_{p}^{*}$ and $O_{p}^{*}$ and are provided in Lemma 3.1 of Chang and Park (1999).

For a sequence of bootstrapped statistics $\left(Z_{n}^{*}\right)$ which weakly converges a.s. (or in $\mathbf{P}$ ), it follows that $Z_{n}^{*}=O_{p}^{*}(1)$ a.s. (or in $\mathbf{P}$ ). Moreover, if $Z_{n}^{*} \rightarrow d^{*} Z$ a.s. (or in $\mathbf{P}$ ), then $Z_{n}^{*}+Y_{n}^{*} \rightarrow_{d^{*}} Z$ a.s. (or in $\mathbf{P}$ ) for any $\left(Y_{n}^{*}\right)$ such that $Y_{n}^{*}=o_{p}^{*}(1)$ a.s. (or in $\mathbf{P}$ ). For further discussions on bootstrap asymptotics, the reader is referred to Park (1999) and Chang and Park (1999).

Lemma A.3 Under Assumption 2.1, we have

(a) $\frac{1}{\sqrt{n}} \sum_{t=1}^{[n s]} w_{t}^{*} \rightarrow_{d}^{*} \Psi(1) B_{\eta}(s)$

(b) $\frac{1}{\sqrt{n}} \sum_{t=1}^{[n s]} u_{t}^{*} \rightarrow_{d}^{*} \pi(1)\left(\beta(1)^{\prime} \Psi(1) B_{\eta}(s)+B_{\varepsilon}(s)\right)$

as $n \rightarrow \infty$.

Proof of Lemma A.3 Under Assumption 2.1, the following invariance principle for $\left(\xi_{t}^{*}\right)=$ $\left(\varepsilon_{t}^{*}, \eta_{t}^{* \prime}\right)^{\prime}$ holds:

$$
\frac{1}{\sqrt{n}} \sum_{t=1}^{[n s]} \xi_{t}^{*} \rightarrow_{d}^{*} B=\left(\begin{array}{c}
B_{\varepsilon} \\
B_{\eta}
\end{array}\right)
$$

as $n \rightarrow \infty$ due to Theorem 3.2 of Chang, Park and Song (2000). As in the proof of Lemma A.1, we use the BN representations for the bootstrapped series $\left(w_{t}^{*}\right),\left(v_{t}^{*}\right)$ and $\left(u_{t}^{*}\right)$ to derive the limit distributions of their partial sum processes.

Part (a) Let $\tilde{\Phi}_{n}(1)=I_{m}-\sum_{k=1}^{\ell} \tilde{\Phi}_{k}$, where $\tilde{\Phi}_{k}$ 's are the coefficient estimates from the fitted regression (15), and define

$$
\tilde{\Psi}_{n}(1)=\tilde{\Phi}_{n}(1)^{-1}
$$

We may derive the $\mathrm{BN}$ representations for $\left(w_{t}^{*}\right)$ from the fitted autoregression (15) as we did for $\left(w_{t}\right)$ in $(28)$ as

$$
w_{t}^{*}=\tilde{\Psi}_{n}(1) \eta_{t-r-1}^{*}+\left(\bar{w}_{t-1}^{*}-\bar{w}_{t}^{*}\right),
$$

where $\bar{w}_{t}^{*}=\sum_{k=1}^{\ell}\left(\tilde{\Psi}_{n}(1) \sum_{j=k}^{\ell} \tilde{\Phi}_{j}\right) w_{t-k+1}^{*}$. Then the stated result in part (a) follows directly 
from the invariance principle (31) as

$$
\frac{1}{\sqrt{n}} \sum_{t=1}^{[n s]} w_{t}^{*}=\tilde{\Psi}_{n}(1) \frac{1}{\sqrt{n}} \sum_{t=1}^{[n s]} \eta_{t-r-1}^{*}+\frac{1}{\sqrt{n}}\left(\bar{w}_{0}^{*}-\bar{w}_{[n s]}^{*}\right) \rightarrow_{d}^{*} \Psi(1) B_{\eta}(s)
$$

as $n \rightarrow \infty$, since $\tilde{\Psi}_{n}(1) \rightarrow a . s . \Psi(1)$ and $n^{-1 / 2}\left(\bar{w}_{0}^{*}-\bar{w}_{[n s]}^{*}\right)=o_{p}^{*}(1)$.

Part (b) Define

$$
\tilde{\pi}_{n}(1)=\tilde{\alpha}_{n}(1)^{-1} \text { and } \tilde{\beta}_{n}(1)=\sum_{k=-r}^{q} \tilde{\beta}_{k},
$$

where $\tilde{\alpha}_{n}(1)$ is defined as in $(22)$ and $\tilde{\beta}_{k}$ 's are the estimates from (14). Now we derive the $\mathrm{BN}$ representation for $\left(u_{t}^{*}\right)$ from the fitted regression (14) similarly as in (29) as

$$
u_{t}^{*}=\tilde{\pi}_{n}(1)\left(\tilde{\beta}_{n}(1)^{\prime} w_{t}^{*}+\varepsilon_{t}^{*}\right)+\tilde{\pi}_{n}(1)\left(\left(\tilde{w}_{t-1}^{*+}-\tilde{w}_{t}^{*+}\right)+\left(\tilde{w}_{t}^{*-}-\tilde{w}_{t-1}^{*-}\right)\right)+\left(\bar{u}_{t-1}^{*}-\bar{u}_{t}^{*}\right),
$$

where

$$
\begin{aligned}
\bar{u}_{t}^{*} & =\tilde{\pi}_{n}(1) \sum_{k=1}^{p}\left(\sum_{i=k}^{p} \tilde{\alpha}_{i}\right) u_{t-k+1}^{*} \\
\tilde{w}_{t}^{*+} & =\sum_{k=0}^{q-1} \tilde{\beta}_{k}^{+\prime} w_{t-k}^{*} \\
\tilde{w}_{t}^{*-} & =\sum_{k=0}^{r-1} \tilde{\beta}_{k}^{-\prime} w_{t+k+1}^{*}
\end{aligned}
$$

with $\tilde{\beta}_{k}^{+}=\sum_{j=k+1}^{q} \tilde{\beta}_{j}$ and $\tilde{\beta}_{k}^{-}=\sum_{j=k+1}^{r} \tilde{\beta}_{-j}$. Note that $\tilde{\pi}_{n}(1) \rightarrow a . s . \pi(1)$ and $\tilde{\beta}_{n}(1) \rightarrow a . s$. $\beta(1)$. Then the stated result follows as

$$
\begin{aligned}
\frac{1}{\sqrt{n}} \sum_{t=1}^{[n s]} u_{t}^{*}= & \tilde{\pi}(1)\left(\tilde{\beta}(1)^{\prime} \frac{1}{\sqrt{n}} \sum_{t=1}^{[n s]} w_{t}^{*}+\frac{1}{\sqrt{n}} \sum_{t=1}^{[n s]} \varepsilon_{t}^{*}\right)+o_{p}^{*}(1) \\
\rightarrow_{d}^{*} & \pi(1)\left(\beta(1)^{\prime} \Psi(1) B_{\eta}(s)+B_{\varepsilon}(s)\right)
\end{aligned}
$$

due to (31) and the result in part (a).

Lemma A.4 Under Assumption 2.1, we have

(a) $n^{-2} \sum_{t=1}^{n} y_{t-1}^{* 2} \rightarrow_{d}^{*} \pi(1)^{2} \int_{0}^{1} Q(s)^{2} d s$

(b) $n^{-1} \sum_{t=1}^{n} y_{t-1}^{*} \varepsilon_{t}^{*} \rightarrow_{d}^{*} \sigma_{\varepsilon} \pi(1) \int_{0}^{1} Q(s) d P(s)$

as $n \rightarrow \infty$, where $Q(s)$ and $P(s)$ are defined in Theorem 2.3.

Proof of Lemma A.4 We have from (19) and the BN representation of $\left(u_{t}^{*}\right)$ derived in (32) that

$$
\frac{1}{\sqrt{n}} y_{[n s]}^{*}=\frac{1}{\sqrt{n}} \sum_{k=1}^{[n s]} u_{k}^{*}+\frac{1}{\sqrt{n}}\left(u_{0}^{*}-u_{[n s]}^{*}\right)+\frac{y_{0}^{*}}{\sqrt{n}}
$$




$$
\begin{aligned}
& =\tilde{\pi}_{n}(1) \frac{1}{\sqrt{n}} \sum_{k=1}^{[n s]}\left(\tilde{\beta}(1)^{\prime} w_{k}^{*}+\varepsilon_{k}^{*}\right)+o_{p}(1)^{*} \\
& \rightarrow_{d}^{*} \pi(1)\left(\beta(1)^{\prime} \Psi(1) B_{\eta}(s)+B_{\varepsilon}(s)\right)
\end{aligned}
$$

using the results in Lemma A.3. Now we may easily derive the stated results in parts (a) and (b) as in the proof of Lemma A.2.

Proof of Theorem 3.3 The stochastic orders for the bootstrap sample moments appearing in the definitions of the bootstrap test $S_{n}^{*}$ and $T_{n}^{*}$ are easily obtained

$$
\left|\left(\sum_{t=1}^{n} z_{t}^{*} z_{t}^{* \prime}\right)^{-1}\right|=O_{p}^{*}\left(n^{-1}\right), \quad\left|\sum_{t=1}^{n} z_{t}^{*} \varepsilon_{t}^{*}\right|=O_{p}^{*}\left(n^{1 / 2}\right) \text { and }\left|\sum_{t=1}^{n} y_{t-1}^{*} z_{t}^{* \prime}\right|=O_{p}^{*}(n)
$$

from the results in Lemma 3.3 of Chang and Park (2000). Then we have

$$
\begin{array}{r}
\left|\left(\sum_{t=1}^{n} y_{t-1}^{*} z_{t}^{* \prime}\right)\left(\sum_{t=1}^{n} z_{t}^{*} z_{t}^{* \prime}\right)^{-1}\left(\sum_{t=1}^{n} z_{t}^{*} \varepsilon_{t}^{*}\right)\right| \leq\left|\sum_{t=1}^{n} y_{t-1}^{*} z_{t}^{* \prime}\right|\left|\left(\sum_{t=1}^{n} z_{t}^{*} z_{t}^{* \prime}\right)^{-1}\right|\left|\sum_{t=1}^{n} z_{t}^{*} \varepsilon_{t}^{*}\right|=O_{p}^{*}\left(n^{1 / 2}\right) \\
\left|\left(\sum_{t=1}^{n} y_{t-1}^{*} z_{t}^{* \prime}\right)\left(\sum_{t=1}^{n} z_{t}^{*} z_{t}^{* \prime}\right)^{-1}\left(\sum_{t=1}^{n} z_{t}^{*} y_{t-1}^{*}\right)\right| \leq\left|\sum_{t=1}^{n} y_{t-1}^{*} z_{t}^{* \prime}\right|\left|\left(\sum_{t=1}^{n} z_{t}^{*} z_{t}^{* \prime}\right)^{-1}\right|\left|\sum_{t=1}^{n} z_{t}^{*} y_{t-1}^{*}\right|=O_{p}^{*}(n)
\end{array}
$$

and consequently

$$
\begin{aligned}
n^{-1} A_{n}^{*} & =n^{-1} \sum_{t=1}^{n} y_{t-1}^{*} \varepsilon_{t}^{*}+o_{p}^{*}(1) \\
n^{-2} B_{n}^{*} & =n^{-2} \sum_{t=1}^{n} y_{t-1}^{* 2}+o_{p}^{*}(1) .
\end{aligned}
$$

Note that $\tilde{\alpha}_{n}(1) \rightarrow$ a.s. $\alpha(1)$ and $\tilde{\sigma}_{n}^{2} \rightarrow a . s . \sigma_{\varepsilon}^{2}$. Then it follows from the definitions of $S_{n}^{*}$ and $T_{n}^{*}$, given in (23) and (24), and the results in the previous equation that

$$
\begin{aligned}
& S_{n}^{*}=\frac{n B_{n}^{*-1} A_{n}^{*}}{\tilde{\alpha}_{n}(1)}=\frac{1}{\tilde{\alpha}_{n}(1)}\left(\frac{n^{-1} \sum_{t=1}^{n} y_{t-1}^{*} \varepsilon_{t}^{*}}{n^{-2} \sum_{t=1}^{n} y_{t-1}^{* 2}}\right)+o_{p}^{*}(1) \\
& T_{n}^{*}=\frac{1}{\tilde{\sigma}_{n}}\left(\frac{A_{n}^{*}}{B_{n}^{* 1 / 2}}\right)=\frac{1}{\tilde{\sigma}_{n}}\left(\frac{n^{-1} \sum_{t=1}^{n} y_{t-1}^{*} \varepsilon_{t}^{*}}{\left(n^{-2} \sum_{t=1}^{n} y_{t-1}^{* 2}\right)^{1 / 2}}\right)+o_{p}^{*}(1) .
\end{aligned}
$$

Now the stated limit theories for $S_{n}^{*}$ and $T_{n}^{*}$ follow immediately from Lemma A.4. 


\section{References}

Aizenman, J. (1984), "Modelling deviations from purchasing power parity (PPP)," International Economic Review, 25, 175-191.

Andrews, D.W.K. (1991), "Heteroskedasticity and autocorrelation consistent covariance matrix estimation," Econometrica, 59, 817-858.

Andrews, D.W.K. and M. Buchinsky (1999), "A three-step method for choosing the number of bootstrap repetitions," Econometrica, 68, 23-52.

Basawa, I.V., A.K. Mallik, W.P. McCormick, J.H. Reeves and R.L. Taylor (1991), "Bootstrapping unstable first-order autoregressive processes," Annals of Statistics, 19, 10981101.

Berk, K.N. (1974), "Consistent autoregressive spectral estimates," The Annals of Statistics, 15, 1050-1063.

Brockwell, P.J. and R.A. Davis (1991), Time Series: Theory and Methods, Springer-Verlag: New York.

Chang, Y. (2000), "Bootstrap unit root tests in panels with cross-sectional dependency," Rice University Working Paper 2000-01.

Chang, Y. and J.Y. Park (1999), "A sieve bootstrap for the test of a unit root," Journal of Time Series Analysis, forthcoming.

Chang, Y. and J.Y. Park (2001), "On the asymptotics of ADF tests for unit roots," Rice University Working Paper 2001-02.

Chang, Y., J.Y. Park and K.M. Song (2000), "Bootstrapping cointegrating regressions," Rice University, mimeographed.

Dejong, D.N., J.C. Nankervis, N.E. Savin, and C.H. Whiteman (1992a), "The power problems of unit root tests in time series with autoregressive errors," Journal of Econometrics, 53, 323-343.

Dejong, D.N., J.C. Nankervis, N.E. Savin, and C.H. Whiteman (1992b), "Integration versus trend stationarity in time series," Econometrica, 60, 423-433.

Domowitz, I., and M. El-Gamal (2001) "A consistent nonparametric test of ergodicity for time series with applications," Journal of Econometrics, 102, 365-398.

Flynn, N.A. and J.L. Boucher (1993), "Tests of long-run purchasing power parity using alternative methodologies," Journal of Macroeconomics, 15, 109-122.

Frankel, J. and A. Rose (1996), "A panel project on purchasing power parity: Mean reversion within and between countries," Journal of International Economics, 40, 209-224. 
Hansen, B.E. (1995), "Rethinking the univariate approach to unit root testing: Using covariates to increase power," Econometric Theory, 11, 1148-1171.

MacDonald, R. (1996), "Panel unit root tests and real exchange rates," Economics Letters, $50,7-11$.

Nelson, C.R. and C. Plosser (1982), "Trends and random walks in macroeconomic time series: Some evidence and implications," Journal of Monetary Economics, 10, 139162.

Ng, S. and P. Perron (1995), "Unit root tests in ARMA models with data-dependent methods for the selection of the truncation lag," Journal of the American Statistical Association, 90, 268-281.

O'Connell, P.G.J. (1998), "The overvaluation of purchasing power parity," Journal of International Economics, 44, 1-20.

Oh, K.Y. (1997), "Purchasing power parity and unit root tests using panel data," Journal of International Money and Finance, 43, 313-323.

Papell, D. (1997), "Searching for stationarity: Purchasing power parity under the current float," Journal of International Economics, 43, 313-323.

Park, J.Y. (1999), "An invariance principle for sieve bootstrap in time series," Econometric Theory, forthcoming.

Perron, P. (1988), "Trends and random walks in macroeconomic time series: Further evidence from a new approach," Journal of Economic Dynamics and Control, 12, 297-332.

Perron, P. (1989), "The Great Crash, the oil price shock, and the unit root hypothesis," Econometrica, 57, 1361-1401.

Phillips, P.C.B. (1987), "Toward a unified asymptotic theory for autoregression," Biometrika, $74,535-547$.

Phillips, P.C.B. and V. Solo (1992), "Asymptotics for linear processes," Annals of Statistics, 20, 971-1001.

Said, S.E. and D.A. Dickey (1984), "Testing for unit roots in autoregressive-moving average models of unknown order," Biometrika, 71, 599-608.

Saikkonen, P. (1991), "Asymptotically efficient estimation of cointegration regressions," Econometric Theory, 7, 1-21.

Schotman, P.C. and H.K. van Dijk (1991), "On Bayesian routes to unit roots," Journal of Applied Econometrics, 6, 387-401.

Shiller, R. and P. Perron (1985), "Testing the random walk hypothesis: Power versus frequency of observation," Economics Letters, 18, 381-386. 
Stock, J. and M. Watson (1999), "Business cycle fluctuations in U.S. macroeconomic time series," Handbook of Macroeconomics, edited by J. Taylor and M. Woodford, North Holland, Vol. 1a, 3-64.

Whitt, J.A. (1992), "The long-run behavior of the real exchange rate: A reconsideration," Journal of Money, Credit, and Banking, 14, 72-82. 
Table 1: Finite Sample Sizes for AR Errors

\begin{tabular}{|c|c|c|c|c|c|c|c|c|c|c|c|}
\hline & \multicolumn{5}{|c|}{$\mathrm{ADF}$} & \multicolumn{3}{|l|}{ CADF } & \multicolumn{3}{|c|}{ BCADF } \\
\hline$\beta$ & $\phi$ & $1 \%$ & $5 \%$ & $10 \%$ & $1 \%$ & $5 \%$ & $10 \%$ & $1 \%$ & $5 \%$ & $10 \%$ & $\hat{\rho}^{2}$ \\
\hline 0.000 & 0.000 & 0.013 & 0.056 & 0.103 & 0.012 & 0.053 & 0.103 & 0.012 & 0.055 & 0.114 & 0.991 \\
\hline \multirow[t]{4}{*}{0.800} & 0.800 & 0.014 & 0.063 & 0.112 & 0.021 & 0.075 & 0.132 & 0.012 & 0.047 & 0.100 & 0.369 \\
\hline & 0.500 & 0.011 & 0.052 & 0.101 & 0.014 & 0.061 & 0.118 & 0.010 & 0.048 & 0.100 & 0.587 \\
\hline & -0.500 & 0.009 & 0.051 & 0.101 & 0.012 & 0.061 & 0.112 & 0.010 & 0.054 & 0.103 & 0.771 \\
\hline & -0.800 & 0.009 & 0.047 & 0.088 & 0.013 & 0.058 & 0.111 & 0.011 & 0.056 & 0.111 & 0.871 \\
\hline \multirow[t]{4}{*}{0.500} & 0.800 & 0.009 & 0.043 & 0.087 & 0.014 & 0.069 & 0.127 & 0.009 & 0.048 & 0.093 & 0.478 \\
\hline & 0.500 & 0.010 & 0.051 & 0.100 & 0.013 & 0.062 & 0.115 & 0.010 & 0.054 & 0.107 & 0.726 \\
\hline & -0.500 & 0.011 & 0.047 & 0.098 & 0.013 & 0.050 & 0.098 & 0.011 & 0.049 & 0.099 & 0.886 \\
\hline & -0.800 & 0.010 & 0.046 & 0.089 & 0.010 & 0.051 & 0.105 & 0.010 & 0.052 & 0.110 & 0.921 \\
\hline \multirow[t]{4}{*}{-0.500} & 0.800 & 0.009 & 0.039 & 0.076 & 0.003 & 0.016 & 0.031 & 0.008 & 0.040 & 0.080 & 0.206 \\
\hline & 0.500 & 0.010 & 0.055 & 0.104 & 0.006 & 0.032 & 0.058 & 0.010 & 0.053 & 0.101 & 0.583 \\
\hline & -0.500 & 0.010 & 0.053 & 0.103 & 0.011 & 0.051 & 0.100 & 0.011 & 0.056 & 0.116 & 0.866 \\
\hline & -0.800 & 0.010 & 0.048 & 0.091 & 0.012 & 0.053 & 0.105 & 0.013 & 0.059 & 0.114 & 0.902 \\
\hline \multirow[t]{4}{*}{-0.800} & 0.800 & 0.008 & 0.041 & 0.083 & 0.001 & 0.006 & 0.017 & 0.006 & 0.037 & 0.079 & 0.030 \\
\hline & 0.500 & 0.011 & 0.054 & 0.103 & 0.004 & 0.018 & 0.036 & 0.009 & 0.042 & 0.092 & 0.307 \\
\hline & -0.500 & 0.012 & 0.053 & 0.102 & 0.010 & 0.050 & 0.098 & 0.010 & 0.055 & 0.115 & 0.679 \\
\hline & -0.800 & 0.011 & 0.054 & 0.102 & 0.011 & 0.051 & 0.099 & 0.011 & 0.055 & 0.113 & 0.754 \\
\hline
\end{tabular}

Note: BCADF stands for the bootstrap CADF test. 
Table 2A: Finite Sample Powers for AR Errors $(\alpha=-0.10)$

\begin{tabular}{|c|c|c|c|c|c|c|c|c|c|c|c|}
\hline & \multicolumn{5}{|c|}{$\mathrm{ADF}$} & \multicolumn{3}{|l|}{ CADF } & \multicolumn{3}{|c|}{ BCADF } \\
\hline$\beta$ & $\phi$ & $1 \%$ & $5 \%$ & $10 \%$ & $1 \%$ & $5 \%$ & $10 \%$ & $1 \%$ & $5 \%$ & $10 \%$ & $\hat{\rho}^{2}$ \\
\hline 0.000 & 0.000 & 0.076 & 0.255 & 0.417 & 0.102 & 0.323 & 0.508 & 0.090 & 0.313 & 0.508 & 0.990 \\
\hline \multirow[t]{4}{*}{0.800} & 0.800 & 0.045 & 0.170 & 0.301 & 0.997 & 0.999 & 1.000 & 0.996 & 1.000 & 1.000 & 0.407 \\
\hline & 0.500 & 0.060 & 0.229 & 0.372 & 0.954 & 0.995 & 0.998 & 0.938 & 0.994 & 0.998 & 0.609 \\
\hline & -0.500 & 0.072 & 0.247 & 0.398 & 0.456 & 0.831 & 0.937 & 0.395 & 0.797 & 0.929 & 0.769 \\
\hline & -0.800 & 0.055 & 0.211 & 0.370 & 0.358 & 0.760 & 0.908 & 0.324 & 0.746 & 0.905 & 0.875 \\
\hline \multirow[t]{4}{*}{0.500} & 0.800 & 0.026 & 0.127 & 0.234 & 0.939 & 0.991 & 0.996 & 0.918 & 0.985 & 0.996 & 0.493 \\
\hline & 0.500 & 0.060 & 0.225 & 0.375 & 0.661 & 0.906 & 0.966 & 0.601 & 0.888 & 0.962 & 0.731 \\
\hline & -0.500 & 0.074 & 0.254 & 0.412 & 0.251 & 0.635 & 0.820 & 0.212 & 0.601 & 0.807 & 0.888 \\
\hline & -0.800 & 0.060 & 0.227 & 0.375 & 0.225 & 0.587 & 0.781 & 0.197 & 0.565 & 0.774 & 0.922 \\
\hline \multirow[t]{4}{*}{-0.500} & 0.800 & 0.029 & 0.129 & 0.241 & 0.716 & 0.863 & 0.922 & 0.807 & 0.940 & 0.973 & 0.207 \\
\hline & 0.500 & 0.093 & 0.296 & 0.454 & 0.171 & 0.389 & 0.534 & 0.226 & 0.518 & 0.675 & 0.585 \\
\hline & -0.500 & 0.071 & 0.260 & 0.416 & 0.073 & 0.256 & 0.420 & 0.068 & 0.252 & 0.422 & 0.866 \\
\hline & -0.800 & 0.061 & 0.225 & 0.375 & 0.075 & 0.252 & 0.416 & 0.068 & 0.252 & 0.418 & 0.903 \\
\hline \multirow[t]{4}{*}{-0.800} & 0.800 & 0.031 & 0.132 & 0.240 & 0.961 & 0.989 & 0.994 & 0.981 & 0.995 & 0.997 & 0.027 \\
\hline & 0.500 & 0.075 & 0.254 & 0.418 & 0.447 & 0.685 & 0.793 & 0.581 & 0.821 & 0.900 & 0.310 \\
\hline & -0.500 & 0.077 & 0.258 & 0.423 & 0.086 & 0.275 & 0.417 & 0.082 & 0.284 & 0.441 & 0.678 \\
\hline & -0.800 & 0.067 & 0.248 & 0.416 & 0.079 & 0.259 & 0.411 & 0.072 & 0.261 & 0.423 & 0.754 \\
\hline
\end{tabular}


Table 2B: Finite Sample Powers for AR Errors $(\alpha=-0.05)$

\begin{tabular}{ccccccccccccc}
\hline \multicolumn{1}{c}{ ADF } & \multicolumn{1}{c}{ CADF } & \multicolumn{7}{c}{ BCADF } \\
\hline$\beta$ & $\phi$ & $1 \%$ & $5 \%$ & $10 \%$ & $1 \%$ & $5 \%$ & $10 \%$ & $1 \%$ & $5 \%$ & $10 \%$ & $\hat{\rho}^{2}$ \\
\hline \multirow{2}{*}{0.000} & 0.000 & 0.029 & 0.120 & 0.220 & 0.030 & 0.131 & 0.240 & 0.024 & 0.126 & 0.241 & 0.991 \\
\hline \multirow{2}{*}{0.800} & 0.800 & 0.028 & 0.106 & 0.194 & 0.949 & 0.984 & 0.993 & 0.937 & 0.983 & 0.991 & 0.406 \\
& 0.500 & 0.026 & 0.110 & 0.196 & 0.554 & 0.827 & 0.916 & 0.504 & 0.808 & 0.910 & 0.608 \\
& -0.500 & 0.026 & 0.113 & 0.201 & 0.105 & 0.340 & 0.521 & 0.077 & 0.297 & 0.487 & 0.771 \\
& -0.800 & 0.021 & 0.090 & 0.176 & 0.076 & 0.270 & 0.446 & 0.067 & 0.255 & 0.435 & 0.875 \\
\hline \multirow{2}{*}{0.500} & 0.800 & 0.013 & 0.071 & 0.138 & 0.718 & 0.885 & 0.940 & 0.664 & 0.871 & 0.932 & 0.492 \\
& 0.500 & 0.023 & 0.107 & 0.205 & 0.238 & 0.519 & 0.690 & 0.192 & 0.484 & 0.670 & 0.731 \\
& -0.500 & 0.029 & 0.115 & 0.206 & 0.058 & 0.215 & 0.367 & 0.047 & 0.198 & 0.351 & 0.889 \\
& -0.800 & 0.023 & 0.095 & 0.181 & 0.053 & 0.196 & 0.343 & 0.043 & 0.188 & 0.333 & 0.922 \\
\hline \multirow{2}{*}{-0.500} & 0.800 & 0.012 & 0.060 & 0.121 & 0.334 & 0.537 & 0.638 & 0.451 & 0.681 & 0.790 & 0.206 \\
& 0.500 & 0.035 & 0.134 & 0.232 & 0.052 & 0.158 & 0.262 & 0.078 & 0.248 & 0.385 & 0.584 \\
& -0.500 & 0.029 & 0.112 & 0.208 & 0.028 & 0.112 & 0.204 & 0.026 & 0.113 & 0.212 & 0.867 \\
& -0.800 & 0.022 & 0.096 & 0.186 & 0.027 & 0.107 & 0.202 & 0.026 & 0.109 & 0.208 & 0.903 \\
\hline-0.800 & 0.800 & 0.015 & 0.072 & 0.137 & 0.699 & 0.846 & 0.901 & 0.808 & 0.922 & 0.957 & 0.029 \\
& 0.500 & 0.028 & 0.114 & 0.214 & 0.134 & 0.308 & 0.438 & 0.222 & 0.474 & 0.621 & 0.308 \\
& -0.500 & 0.028 & 0.111 & 0.201 & 0.029 & 0.113 & 0.216 & 0.028 & 0.118 & 0.231 & 0.679 \\
& -0.800 & 0.023 & 0.110 & 0.200 & 0.026 & 0.112 & 0.201 & 0.026 & 0.116 & 0.213 & 0.754 \\
\hline
\end{tabular}




\section{Table 3. Tests for a Unit Root in the Extended Nelson-Plosser Series}

Panel (a): 1930-1988

\begin{tabular}{lcccccccc}
\hline Variable & AIC & BIC & STC & $Z_{t}$ & $Z_{\alpha}$ & CADF & Covariate & $\hat{\rho}^{2}$ \\
\hline Nominal GNP & -2.113 & -2.113 & $-3.245^{c}$ & $-4.447^{a}$ & $-30.338^{a}$ & $-2.398^{b * *}$ & Employment \\
Industrial production & $-5.159^{a}$ & $-5.159^{a}$ & $-5.159^{a}$ & -2.716 & -14.349 & -0.063 & Unemployment rate & 0.012 \\
GNP deflator & -2.021 & -2.021 & $-4.072^{a}$ & -2.961 & -12.254 & $-3.944^{a * *}$ & Real GNP & 0.009 \\
Consumer prices & $-3.346^{c}$ & -1.280 & -2.016 & -2.646 & -9.741 & $-2.289^{c *}$ & Real GNP & 0.084 \\
Wages & $-3.977^{a}$ & -2.236 & $-3.977^{a}$ & $-4.304^{a}$ & $-26.822^{b}$ & $-4.703^{a * *}$ & Unemployment rate & 0.032 \\
Money stock & -2.717 & -2.717 & -2.717 & $-3.551^{b}$ & $-23.166^{b}$ & $-2.779^{b * *}$ & Unemployment rate & 0.003 \\
Velocity & -2.466 & $-4.348^{a}$ & -2.925 & $-4.579^{a}$ & $-35.449^{a}$ & $-7.906^{a * *}$ & Employment & 0.002 \\
Interest rate & $-3.411^{b}$ & $-3.411^{b}$ & -2.496 & -2.624 & -7.227 & $-3.081^{a * *}$ & Employment & 0.000 \\
Common stock prices & -1.727 & -1.727 & $-3.361^{c}$ & $-4.214^{b}$ & $-20.432^{c}$ & $-3.187^{a * *}$ & Real GNP \\
\hline
\end{tabular}

Panel (b): 1930-1972

\begin{tabular}{lcccccccc}
\hline Variable & AIC & BIC & STC & $Z_{t}$ & $Z_{\alpha}$ & CADF & Covariate & $\hat{\rho}^{2}$ \\
\hline Industrial production & $-6.062^{a}$ & $-6.062^{a}$ & $-6.062^{a}$ & -3.057 & -16.460 & $-4.703^{a * * *}$ & Unemployment rate & 0.045 \\
Real wages & $-3.355^{c}$ & $-3.355^{c}$ & -3.097 & $-3.203^{c}$ & $-20.122^{c}$ & $-6.603^{a * * *}$ & Real per capita GNP & 0.070 \\
\hline
\end{tabular}

Panel (c):1930-1988

\begin{tabular}{lccccc}
\hline Covariate & AIC & BIC & STC & $Z_{t}$ & $Z_{\alpha}$ \\
\hline Real GNP & $-5.253^{a}$ & $-3.307^{c}$ & $-3.547^{b}$ & $-3.424^{c}$ & $-22.870^{b}$ \\
Real per capita GNP & $-6.557^{a}$ & $-6.557^{a}$ & $-6.557^{a}$ & $-3.295^{c}$ & $-21.230^{b}$ \\
Employment & $-4.866^{a}$ & $-3.161^{c}$ & $-4.866^{a}$ & $-3.802^{b}$ & $-27.166^{b}$ \\
Unemployment rate & $-3.065^{b}$ & $-3.065^{b}$ & $-3.003^{b}$ & $-2.692^{c}$ & $-13.996^{b}$ \\
Real wages & -1.222 & -1.222 & -1.302 & -1.337 & -5.169 \\
\hline
\end{tabular}

Note.

1. Columns 2-4 denote the ADF test results using the lag order selection criteria such as AIC, BIC, and STC (the sequential test criterion).

2. The result for unemployment rate series is obtained without a time trend in the regression.

3. a, b, and c denote statistical significance at the $1 \%, 5 \%$, and $10 \%$ level, respectively.

4. $* * * * *$, and $*$ denote statistical significance at the $1 \%, 5 \%$, and $10 \%$ level, respectively, for the bootstrap test. 
Table 4. Tests for a Unit Root in Real Exchange Rates

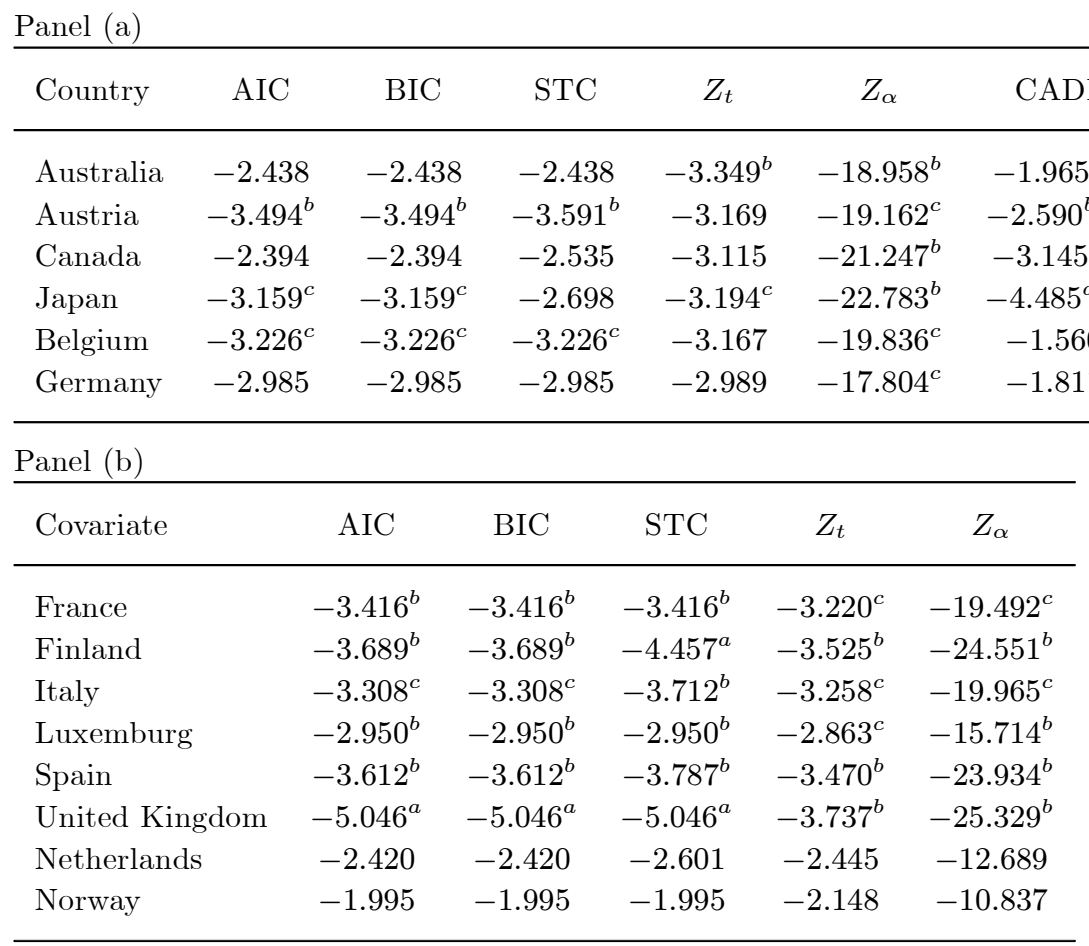

Note.

1. Columns 2-4 denote the ADF test results using the lag order selection criteria such as AIC, BIC, and STC (the sequential test criterion).

2. The result for Luxemburg with the ADF test and those for Australia, Austria, and Belgium with the CADF test are obtained without including a time trend function.

3. See Note in Table 3 for the definition of $(\mathrm{a}, \mathrm{b}, \mathrm{c})$ and $(* * *, * *, *)$. 